\title{
Diffusional and accretional growth of water drops in a rising adiabatic parcel: effects of the turbulent collision kernel
}

\author{
W. W. Grabowski ${ }^{1}$ and L.-P. Wang ${ }^{2}$ \\ ${ }^{1}$ Mesoscale and Microscale Meteorology Division, National Center for Atmospheric Research, Boulder, CO 80307, USA \\ ${ }^{2}$ Department of Mechanical Engineering, University of Delaware, Newark, DE 19716, USA
}

Received: 26 June 2008 - Published in Atmos. Chem. Phys. Discuss.: 31 July 2008

Revised: 20 November 2008 - Accepted: 24 March 2009 - Published: 2 April 2009

\begin{abstract}
A large set of rising adiabatic parcel simulations is executed to investigate the combined diffusional and accretional growth of cloud droplets in maritime and continental conditions, and to assess the impact of enhanced droplet collisions due to small-scale cloud turbulence. The microphysical model applies the droplet number density function to represent spectral evolution of cloud and rain/drizzle drops, and various numbers of bins in the numerical implementation, ranging from 40 to 320 . Simulations are performed applying two traditional gravitational collection kernels and two kernels representing collisions of cloud droplets in the turbulent environment, with turbulent kinetic energy dissipation rates of 100 and $400 \mathrm{~cm}^{2} \mathrm{~s}^{-3}$. The overall result is that the rain initiation time significantly depends on the number of bins used, with earlier initiation of rain when the number of bins is low. This is explained as a combination of the increase of the width of activated droplet spectrum and enhanced numerical spreading of the spectrum during diffusional and collisional growth when the number of model bins is low. Simulations applying around 300 bins seem to produce rain at times which no longer depend on the number of bins, but the activation spectra are unrealistically narrow. These results call for an improved representation of droplet activation in numerical models of the type used in this study.

Despite the numerical effects that impact the rain initiation time in different simulations, the turbulent speedup factor, the ratio of the rain initiation time for the turbulent collection kernel and the corresponding time for the gravitational kernel, is approximately independent of aerosol characteristics, parcel vertical velocity, and the number of bins used in the numerical model. The turbulent speedup factor is in the range $0.75-0.85$ and $0.60-0.75$ for the turbulent kinetic energy dissipation rates of 100 and $400 \mathrm{~cm}^{2} \mathrm{~s}^{-3}$, respectively.
\end{abstract}

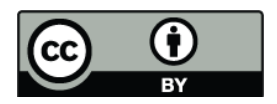

Correspondence to: W. W. Grabowski (grabow@ucar.edu)

\section{Introduction}

Development of drizzle and rain in warm ice-free clouds, the so-called warm-rain process, has been a subject of vigorous research over past several decades (e.g., Pruppacher and Klett, 1997; see the introduction section in Xue et al., 2008 hereafter XWG08 - for a review of issues relevant to the current study). It is clear from these studies that growth of small cloud droplets (radii smaller than about $20 \mu \mathrm{m}$ ) is mostly due to diffusion of water vapor, whereas formation of drizzle and rain (radii larger than $100 \mu \mathrm{m}$ ) involves collisions between drops and their subsequent coalescence. Gravitational collisions (i.e., collisions resulting from different sedimentation velocities of droplets with different sizes) form a basis of the classical model of warm-rain formation. However, there is circumstantial evidence that rain in nature may form more rapidly than predicted by such a model (see discussion in XWG08) and the effects of turbulence on gravitational collisions are often argued to be the factor accelerating warm rain formation (e.g., Pinsky and Khain, 1997, 2002; Falkovich et al., 2002; Ghosh et al., 2005; Riemer and Wexler, 2005; Wang et al., 2006).

XWG08 investigated warm rain formation through collision-coalescence using various formulations of the collection kernel and focusing on the enhancement of the gravitational collision-coalescence due to small-scale turbulence. However, XWG08's theoretical study considered only collisional growth and their calculations were initiated using a prescribed cloud droplet spectra. The impact of cloud turbulence using the most realistic turbulent collection kernel, the Ayala kernel (Ayala, 2008a, b), was shown to be significant, reducing the time by a few tens of percent for high turbulence intensity. Studies applying more realistic droplet growth conditions, including droplet activation, diffusional growth, and eventual collision-coalescence, are needed to assess the impact of cloud turbulence on warm rain development with more confidence. Arguably, the ultimate goal

Published by Copernicus Publications on behalf of the European Geosciences Union. 
should be to investigate this problem using a cloud model, where cloud microphysics can interact in a realistic manner with cloud dynamics, and where development of rain can be directly compared to cloud observations. Before such studies are undertaken, however, one should test the microphysical scheme in a more idealized framework to ensure that microphysical predictions are robust and do not depend, for instance, on details of the numerical grid, model time step, or representation of various microphysical processes. This paper reports on exactly such simulations applying the highly idealized framework of an adiabatic rising parcel model.

One may also consider effects of turbulence on the condensational growth of cloud droplets. In general, turbulence can potentially widen the droplet spectrum and thus subsequently promote the growth due to collision-coalescence. Such effects, however, remain unclear for adiabatic parts of convective clouds. Direct numerical simulations (DNS) described in Vaillancourt et al. (2002) suggest that the impact of small-scale turbulence on the width of droplet spectrum is insignificant because fluctuations of the supersaturation are small and small-scale turbulence combined with droplet sedimentation rearranges droplets rapidly. As a result, the Lagrangian histories of droplet growth, the key parameter determing the width of the droplet spectrum (Cooper, 1989) differ little. Shaw et al. (1998) argued that the presence of coherent structures (vortex tubes) in high-Reynolds-number cloud turbulence, beyond the range accessible to DNS, may result in cloud volumes void of cloud droplets for extended periods of time and even result in additional droplet activation above the cloud base. However, their analysis neglected droplet sedimentation, the key process at the cloud microscale (see Grabowski and Vaillancourt, 1999 for a comment to Shaw et al., 1998). Entrainment and mixing can significantly impact the width of cloud droplet spectra, but this effect is irrelevant for adiabatic parts of a cloud considered here.

The next section discusses formulation of the model and its numerical implementation. Section 3 presents formulation of gravitational and turbulent collection kernels. Results are presented in Sect. 4 and additional sensitivity simulations are reviewed in Sect. 5. A brief discussion of model results in Sect. 6 concludes the paper.

\section{Adiabatic parcel model}

The model solves equations describing conservation of the moist static energy and total water in a rising adiabatic parcel, with the pressure of the parcel assumed equal to the environmental pressure at each height. These can be written as time $(t)$ evolution equations for the temperature $T$, water vapor mixing ratio $q_{v}$, air pressure $p$, and the spectral density function $\phi(r)$ of cloud drops [where $\phi(r) \equiv d n(r) / d r, d n(r)$ is the concentration, per unit mass of dry air, of drops in the radius interval $(r, r+d r)]$. The equations are as follows:

$c_{p} \frac{d T}{d t}=-g w+L C$

$\frac{d q_{v}}{d t}=-C$

$\frac{d p}{d t}=-\rho_{o} w g$

$\frac{\partial \phi}{\partial t}+\frac{\partial}{\partial r}\left(\frac{d r}{d t} \phi\right)=\left(\frac{\partial \phi}{\partial t}\right)_{\text {act }}+\left(\frac{\partial \phi}{\partial t}\right)_{\text {coal }}$

where $g$ is the acceleration of gravity; $w$ is the prescribed vertical velocity of the rising parcel; $L=2.5 \times 10^{6} \mathrm{~J} \mathrm{~kg}^{-1}$ is the latent heat of vaporization; $c_{p}=1005 \mathrm{~J} \mathrm{~kg}^{-1} \mathrm{~K}^{-1}$ is the specific heat of air at constant pressure; $C$ is the condensation rate related to the second term on the left-hand-side of (1d) that represents growth of cloud drops by the condensation of water vapor (the advection of $\phi$ in the radius space; $d r / d t$ is the rate of change of the droplet radius $r$ due to condensation of water vapor); $\rho_{o}=1 \mathrm{~kg} \mathrm{~m}^{-3}$ is the reference air density; and the two terms of the right-hand-side of (1d) represent sources due to cloud droplet activation (i.e., the initial source of cloud droplets) and changes of the spectral density function due to collision-coalescence. Note that assuming constant air density in (1c) instead of an ambient density profile simplifies the governing equations; this is equivalent to the so-called shallow convection approximation valid when the parcel vertical displacement is much smaller than the atmospheric density scale height (equal to about $8 \mathrm{~km}$ ). Note that (1d) assumes that droplets and drops remain inside the rising parcel. Such an approximation is accurate for the cloud droplets (sedimentation velocities below $10 \mathrm{~cm} \mathrm{~s}^{-1}$ ), but is inappropriate for raindrops (sedimentation velocities of a few $\mathrm{m} \mathrm{s}^{-1}$ ). However, the same comment applies to even more idealized simulations described in XWG08 and this aspect needs to be kept in mind when comparing model results to estimates of rain formation time in natural clouds.

The condensation rate $C$ is given by:

$$
\begin{aligned}
C & \equiv \int q^{(0)}\left(\frac{\partial \phi}{\partial t}\right)_{\text {cond }} d r \\
& =\int q^{(0)}\left[-\frac{\partial}{\partial r}\left(\frac{d r}{d t} \phi\right)\right] d r
\end{aligned}
$$

where $q^{(0)}=4 / 3 \pi \rho_{w} r^{3}$ is the mass of a single drop with radius $r\left(\rho_{w}=10^{3} \mathrm{~kg} \mathrm{~m}^{-3} \text { is the water density }\right)^{1}$. The condensed water mixing ratio is $q_{c} \equiv \int q^{(0)} \phi d r$.

The rate of change of the drop radius $r$ due to condensation of water vapor is given by $d r / d t=f_{\text {vent }} A S / r$,

\footnotetext{
${ }^{1}$ For clarity, (2) neglects condensation associated with the initial activation of cloud droplets in (1d). This (negligible) source of cloud condensate and associated latent heating is included in the numerical model.
} 
where $f_{\text {vent }}$ is the ventilation coefficient (i.e., the enhancement factor of the condensational growth rate or evaporation for a drop falling at terminal velocity in comparison to the motionless drop), $A \approx 10^{-10} \mathrm{~m}^{2} \mathrm{~s}^{-1}$, and $S$ is the supersaturation. The ventilation effects are modeled in a standard way (cf. (13.60) and (13.61) in Pruppacher and Klett, 1997). These effects are important for raindrop evaporation and are negligible for growth of drops considered here. The supersaturation is calculated as $S \equiv q_{v} / q_{v s}-1$, where $q_{v s}=\epsilon e_{s}(T) /\left[p-e_{s}(T)\right]$ is the saturated water vapor mixing ratio, $e_{S}(T)=e_{00} \exp \left[L / R_{v}\left(1 / T_{00}-1 / T\right)\right]$ is the water vapor pressure at saturation; $\epsilon=R_{d} / R_{v} ; R_{d}=287 \mathrm{~J} \mathrm{~kg}^{-1} \mathrm{~K}^{-1}$ and $R_{v}=461 \mathrm{~J} \mathrm{~kg}^{-1} \mathrm{~K}^{-1}$ are the gas constants for the dry air and water vapor, respectively; $T_{00}=283.16 \mathrm{~K}$ and $e_{00}=1227 \mathrm{~Pa}$ are the reference values of the temperature and saturated water vapor pressure around which the Clausius-Clapeyron relationship is applied with $L=$ const.

In the discrete system consisting of $\mathcal{N}$ bins (or classes) of drop sizes, the spectral density function for each bin (i) (radius $r^{(i)}$ ) is defined as $\phi^{(i)}=n^{(i)} / \Delta r^{(i)}$, where $n^{(i)}$ is the concentration (per unit mass) of drops in the bin $i$, $\Delta r^{(i)}=r^{(i+1 / 2)}-r^{(i-1 / 2)}$ is the width of this bin, and the bin boundaries are defined as $r^{(i+1 / 2)}=0.5\left(r^{(i+1)}+r^{(i)}\right)$. This transforms the continuous Eq. (1d) into a system of $\mathcal{N}$ coupled equations:

$$
\begin{array}{r}
\frac{\partial \phi^{(i)}}{\partial t}=\left(\frac{\partial \phi^{(i)}}{\partial t}\right)_{\mathrm{cond}}+\left(\frac{\partial \phi^{(i)}}{\partial t}\right)_{\mathrm{act}}+\left(\frac{\partial \phi^{(i)}}{\partial t}\right)_{\mathrm{coal}}, \\
\text { for } i=1, \ldots, \mathcal{N}
\end{array}
$$

where the first term on the right-hand-side represents the condensational growth term in (1d) (i.e., the transport of droplets from one bin to another due to their growth by diffusion of water vapor) and, as in (1d), the second and the third term represent cloud droplet activation and growth by collisioncoalescence. The cloud water mixing ratio in the discrete system is given by $q_{c}=\sum_{i=1}^{\mathcal{N}} q_{i}^{(0)} \phi^{(i)} \Delta r^{(i)}$, where $q_{i}^{(0)}$ is the mass of a single droplet with radius $r^{(i)}$.

The activation term in (3) represents the initial source of cloud droplets due to activation of cloud condensation nuclei (CCN). As in many other detailed microphysics models (e.g., Clark, 1974; Hall, 1980; Grabowski, 1989; Stevens et al., 1996), it is assumed that activated droplets are added to the first size bin. The number of activated CCN $N_{\mathrm{CCN}}$ is related to the supersaturation $S$ through a traditional expression (e.g., Twomey, 1959; see also Pruppacher and Klett, 1997):

$N_{\mathrm{CCN}}=C_{0}(100 S)^{k}$

where $C_{0}$ and $k$ are coefficients determined by the characteristics of the CCN. Herein, we contrast the clean maritime conditions (hereafter MARITIME) and polluted continental conditions (hereafter CONTINENTAL) by assuming $C_{0}=120(\mathrm{mg})^{-1}$ and $k=0.4$ for the MARITIME case and $C_{0}=1000(\mathrm{mg})^{-1}$ and $k=0.6$ for the CONTINENTAL case $^{2}$. Equation (4) is used in the model in the following way. At every time step, the value of the predicted supersaturation $S$ is compared to the maximum supersaturation $S_{\max }$ experienced by the parcel in the past ( $S_{\max }$ is tracked by the model). If $S>S_{\max }$, then additional condensation nuclei have to be activated and their number is derived as $\Delta n=C_{0}(100 S)^{k}-C_{0}\left(100 S_{\max }\right)^{k}$. Subsequently, the spectral density function in the first bin is increased by $\Delta n / \Delta r^{(1)}$ and $S_{\max }$ takes the value of $S$. Such a simple approach, commonly used in numerical models with bin microphysics, results in realistic predictions of the nucleated number of cloud droplets, but not necessary spectral characteristics of cloud droplet spectrum after activation. This aspect will play a significant role in the discussion of model results presented in this paper.

The numerical treatment of the coalescence term is the same as in Morrison and Grabowski (2007). In general, this term can be expressed as a difference between the source term representing collisions of two droplets from different bins that result in formation of a droplet in bin $(i)$ and the sink term representing collisions of droplets from bin $(i)$ with all other droplets (e.g., Pruppacher and Klett, 1997). For the spectral (number) density function $\phi$ represented using a finite number of bins, the coalescence term can be schematically written as:

$\left(\frac{\partial \phi^{(i)}}{\partial t}\right)_{\text {coal }}=\sum_{k=1}^{i} K_{k l} \phi^{(k)} \phi^{(l)}-\phi^{(i)} \sum_{k=1}^{\mathcal{N}} K_{k i} \phi^{(k)}$,

where the first sum is only for bins $k$ and $l$ such that two colliding drops from these bins create a droplet from the bin $i$ (i.e., $q_{k}^{(0)}+q_{l}^{(0)}$ falls within bin $i$ ), and $K_{k l}$ is the collection kernel for droplets from bins $k$ and $l$. In the numerical implementation, we consider binary collisions between drops from all bins, and move resulting drops into appropriate target bins. This ensures the exact conservation of the total mass of the condensed water and the correct change of the number of drops during collisional growth. The Linear Flux Method of Bott (1998) is used in the calculations ${ }^{3}$.

The system (1a, b, c) and (3) is solved using the time splitting technique, with condensational and collisional growth calculated with different frequencies. Collisional growth is calculated using a forward-in-time approach on longer time steps (between 0.5 and $2 \mathrm{~s}$ ), whereas condensation (treated as advection in the radius space using the $1 \mathrm{D}$ advection scheme of Smolarkiewicz, 1984) applies a centered-in-time

\footnotetext{
${ }^{2}$ Note that the coefficient $C_{0}$ as well as the drop concentrations are expressed in this paper in units of number per mg of dry air or simply $(\mathrm{mg})^{-1}$. This is because the numerical values in these units correspond to the concentrations per unit volume expressed in $\mathrm{cm}^{-3}$ when the air density is $1 \mathrm{~kg} \mathrm{~m}^{-3}$. In cloud physics, concentrations of cloud droplets are typically expressed in units of $\mathrm{cm}^{-3}$.

${ }^{3}$ Application of the Bott's approach implies that the exact conservation of drop numbers is no longer satisfied, but the total mass is conserved.
} 
Table 1. Grid formulation parameters and time steps for collisional $\left(\Delta t_{\text {coll }}\right)$ and condensational $\left(\Delta t_{\text {cond }}\right)$ growth for the case of $w=1 \mathrm{~m} \mathrm{~s}^{-1}$.

\begin{tabular}{lcccc}
\hline Eq. (6) & & & & \\
\hline $\mathcal{N}$ & $\alpha$ & $\beta$ & $\Delta t_{\text {coll }}$ & $\Delta t_{\text {cond }}$ \\
69 & 0.25 & 0.055 & $1 \mathrm{~s}$ & $0.2 \mathrm{~s}$ \\
120 & 0.125 & 0.032 & $1 \mathrm{~s}$ & $0.2 \mathrm{~s}$ \\
200 & 0.075 & 0.019 & $0.5 \mathrm{~s}$ & $0.1 \mathrm{~s}$ \\
300 & 0.05 & 0.0125 & $0.2 \mathrm{~s}$ & $0.05 \mathrm{~s}$ \\
\hline Eq. (7) & & & & \\
\hline $\mathcal{N}$ & $\alpha$ & $s$ & $\Delta t_{\text {coll }}$ & $\Delta t_{\text {cond }}$ \\
40 & 1.0 & 1 & $2 \mathrm{~s}$ & $0.5 \mathrm{~s}$ \\
80 & 0.5 & 2 & $1 \mathrm{~s}$ & $0.5 \mathrm{~s}$ \\
160 & 0.25 & 4 & $1 \mathrm{~s}$ & $0.5 \mathrm{~s}$ \\
320 & 0.125 & 8 & $0.5 \mathrm{~s}$ & $0.1 \mathrm{~s}$ \\
\hline
\end{tabular}

predictor-corrector technique using shorter time steps (between 0.05 and $0.5 \mathrm{~s}$ ). To ensure that numerical results are not affected by the details of the finite difference setup, we apply several grid configurations in the radius space and various time stepping intervals. All grids follow a general strategy, already applied in Morrison and Grabowski (2007), where the grid spacing is close to uniform in the range dominated by the diffusional growth (say, for $r$ smaller than $20 \mu \mathrm{m}$ ) and rapidly increases in the collisional growth range to allow covering the needed range (say, up to $10 \mathrm{~mm}$ ) with a reasonable number of bins. Morrison and Grabowski (2007) applied the linear-exponential grid, with the mean radius $r_{i}$ (in $\mu \mathrm{m}$ ) for each bin $i$ given by:

$r_{i}=(i-1) \alpha+10^{(i-1) \beta} \quad$ for $i=1, \ldots, \mathcal{N}$,

where $\alpha$ and $\beta$ are parameters (see Table 1). We also apply a linear-mass doubling grid which combines the linear grid with a grid (often used in collision/coalescence studies) where the drop mass doubles every $s$ bins. In this case the radius $r_{i}$ (in $\mu \mathrm{m}$ ) is obtained as:

$r_{i}=(i-1) \alpha+\left(\frac{3 m_{i}}{4 \pi \rho_{w}}\right)^{1 / 3}$ for $i=1, \ldots, \mathcal{N}$,

where the mass $m_{i}$ is given by the recurrence $m_{i} / m_{i-1}=2^{1 / s}$ and $m_{0}$ is taken as the mass of a droplet with $1-\mu \mathrm{m}$ radius. Note that the second term on rhs of (7) needs to be converted into microns before it is added to the first term. Table 1 shows the grid parameters $(N, \alpha, \beta$, and $s$, as well as model time steps applied in simulations with $w=1 \mathrm{~m} \mathrm{~s}^{-1}$ ) for 8 grid configurations applied in this study. Figure 1 illustrates the grids.

Initial conditions for all simulations are $T(0)=288.16 \mathrm{~K}$, $p(0)=900 \mathrm{hPa}, q_{v}(0)=q_{v s}[T(0), p(0)]$ (i.e., $S(0)=0$ ), and $\phi^{(i)}(0)=0$ for $i=1, \ldots, \mathcal{N}$. Most of the simulations are performed assuming parcel vertical velocity of $w=1 \mathrm{~m} \mathrm{~s}^{-1}$, and

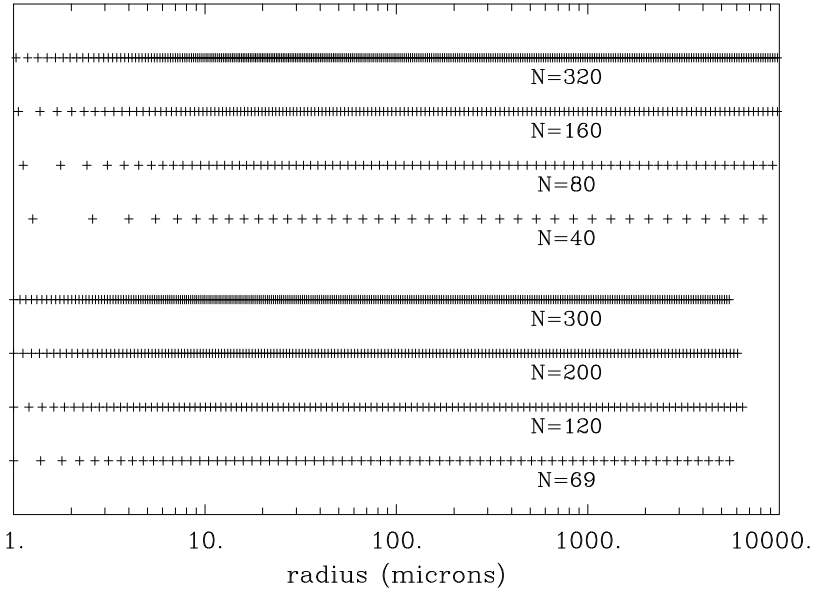

Fig. 1. Grid configurations used in this study. The lower four are for (6) and the upper four are for (7). See text for details.

some simulations using $w=0.2$ and $w=5 \mathrm{~m} \mathrm{~s}^{-1}$ will be reported as well. The calculations proceed until the radar reflectivity factor (the sixth moment of the droplet size distribution) reaches $30 \mathrm{dBz}$.

\section{Formulation of collection kernels}

The impact of turbulent collisions on warm rain initiation is evaluated by comparing results obtained using the turbulent collection kernel with the classical gravitational kernel. Since gravitational kernel may differ to some degree due to different formulations of drop terminal velocity or collision efficiency, we select two formulations of the gravitational kernel.

The gravitational collection kernel without effects of turbulence is given by:

$K_{i j}=E_{i j}^{g} \pi\left(r_{i}+r_{j}\right)^{2}\left|v_{i}^{t}-v_{j}^{t}\right|$,

where $E_{i j}^{g}$ is the collision efficiency of droplets with radii $r_{i}$ and $r_{j}$ in a quiescent background air, and $v_{i}^{t}$ and $v_{j}^{t}$ are their sedimentation (terminal) velocities. Two formulations of the collision efficiencies and terminal velocities are used here. The first one follows that of Long (1974) as given in Simmel et al. (2002; see Sect. 4.2.1 therein) and it will be referred to as the Long kernel. The second one applies tabulated collision efficiencies given in Hall (1980) and terminal velocities of Beard (1976) as given by Pruppacher and Klett (1997). This kernel will be referred to as the Hall kernel.

The turbulent collection kernel employed in this paper combines the analytical parameterization of turbulent geometric collection kernel of Ayala et al. (2008b) with the collision-efficiency enhancement factor obtained from a hybrid direct numerical simulation (Wang et al., 2008). 
Namely, the turbulent collection kernel is expressed as

$K_{i j}=K_{i j}^{0} E_{i j}^{g} \eta_{E}$

where the turbulent geometric collection kernel $K_{i j}^{0}$ is obtained when droplet-droplet local aerodynamic interaction is not considered, in which case the disturbance flows induced by other droplets are excluded when the motion of a given droplet is solved. The collision efficiency of droplets in a quiescent background air $E_{i j}^{g}$ is as in (8). The ratio of turbulent collision efficiency to $E_{i j}^{g}$ is represented by the relative enhancement factor $\eta_{E}$, see Wang et al. (2005). The geometric collection kernel $K_{i j}^{0}$ is given by the following kinematic formulation (Wang et al., 2005)

$K_{i j}^{0}=2 \pi R^{2}\left\langle\left|w_{r}(r=R)\right|\right\rangle g_{i j}(r=R)$,

where the radial relative velocity $w_{r}$ is defined in terms of the center-to-center separation vector $\mathbf{r}$ (pointing from a droplet of radius $r_{j}$ to a droplet of radius $r_{i}$ ), the velocity $\mathbf{V}_{i}$ of the $r_{i}$ droplet, and the velocity $\mathbf{V}_{j}$ of the $r_{j}$ droplet as $w_{r}=\mathbf{r} \cdot\left(\mathbf{V}_{i}-\mathbf{V}_{j}\right) / r$ with $r=|\mathbf{r}| . R \equiv r_{i}+r_{j}$ is the geometric collision radius. The additional factor $g_{i j}$ is the radial distribution function which measures the effect of preferential concentration on the pair number density at separation $r=R$. Both $\left\langle\left|w_{r}\right|\right\rangle$ and $g_{i j}$ in (10) are computed without local aerodynamic interaction. The kinematic formulations (9) and (10) have been validated against dynamic collision rates from DNS, for both ghost droplets and aerodynamicallyinteracting droplets in a turbulent air flow, see Wang et al. (2005) and Ayala et al. (2008a).

Ayala et al. (2008b) developed parameterizations for both $\left\langle\left|w_{r}\right|\right\rangle$ and $g_{i j}$, guided by data from DNS. It should be noted that their parameterizations consider the effects of flow Reynolds number which cannot be fully represented by the hybrid DNS. For example, the parameterization for $\left\langle\left|w_{r}\right|\right\rangle$ makes use of velocity correlations that are valid for both the dissipation subrange and the energy-containing subrange of turbulence. The intermittency of small-scale turbulent fluctuations was incorporated into the model for $g_{i j}$ following Chun et al. (2005). The detailed expression for $K_{i j}^{0}$ can be found in Ayala et al. (2008b). The enhancement factor $\eta_{E}$ is interpolated from the hybrid DNS results reported in Wang et al. (2008) and depends on the flow dissipation rate. We apply the Ayala turbulent collection kernel for two dissipation rates, 100 and $400 \mathrm{~cm}^{2} \mathrm{~s}^{-3}$, and refer to these kernels as A100 and A400, respectively.

Figure 2 shows the ratio of the turbulent kernel to the Hall gravitational kernel for both flow dissipation rates. This ratio is a product of the enhancement of geometric kernel by air turbulence and of $\eta_{E}$ (Wang et al., 2008). Several important inferences can be made from Fig. 2. First, a noticeable enhancement occurs for droplets less than $100 \mu \mathrm{m}$. Second, the overall enhancement is moderate with a value ranging from 1.0 to 5.0. The enhancement factors shown in Fig. 2 are similar to those reported recently by Pinsky and Khain (2004)

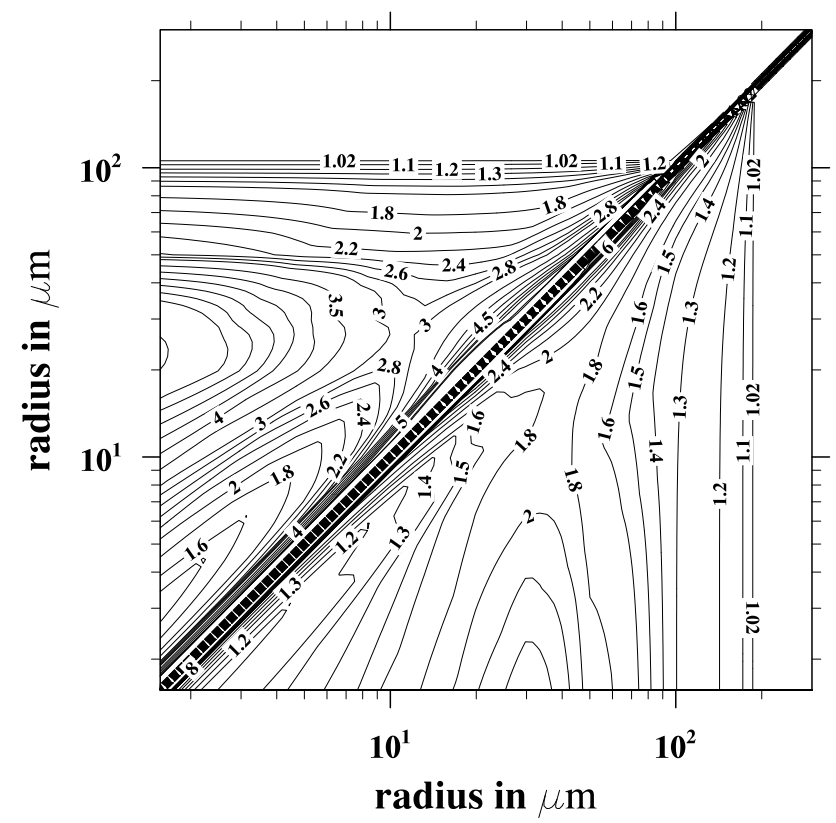

Fig. 2. The ratio of the turbulent collection kernel to the Hall kernel for the flow dissipation rate of 400 and $100 \mathrm{~cm}^{2} \mathrm{~s}^{-3}$ in the upperleft and lower-right part of the figure, respectively. The ratio on the diagonal is undefined due to the zero value of the Hall kernel. The flow rms velocity is assumed $202 \mathrm{~cm} \mathrm{~s}^{-1}$ in both cases.

and Pinsky et al. (2006), where dramatically different approaches were employed. Third, the enhancement is more uniform for droplets less than $60 \mu \mathrm{m}$ than other unrealistic turbulent kernels such as in Riemer and Wexler (2005) for reasons discussed in Wang et al. (2006). Finally, the enhancement is close to one when droplets are above $100 \mu \mathrm{m}$ (an unexpected larger enhancement for the 100-200 $\mu \mathrm{m}$ drop range in the case of A100 comes from the stronger clustering of these drops in comparison to A400 case because the Stokes number, the ratio of droplet inertial response time to the flow Kolmogorov time, is closer to unity for the lower dissipation rate).

\section{Results for CONTINENTAL and MARITIME condi- tions for $\mathrm{w}=1 \mathrm{~m} \mathrm{~s}^{-1}$}

\subsection{Typical evolution of microphysical properties}

We start with a general overview of results obtained using the rising parcel framework, and contrasting the CONTINENTAL and MARITIME conditions. Figures 3 and 4 illustrate the results obtained using the Hall gravitational kernel in the CONTINENTAL $w=1 \mathrm{~m} \mathrm{~s}^{-1}$ case and applying 320 bins. Results for other kernels are qualitatively similar and are not shown. Figure 3 shows the evolution of the temperature, water vapor mixing ratio, condensed cloud water 


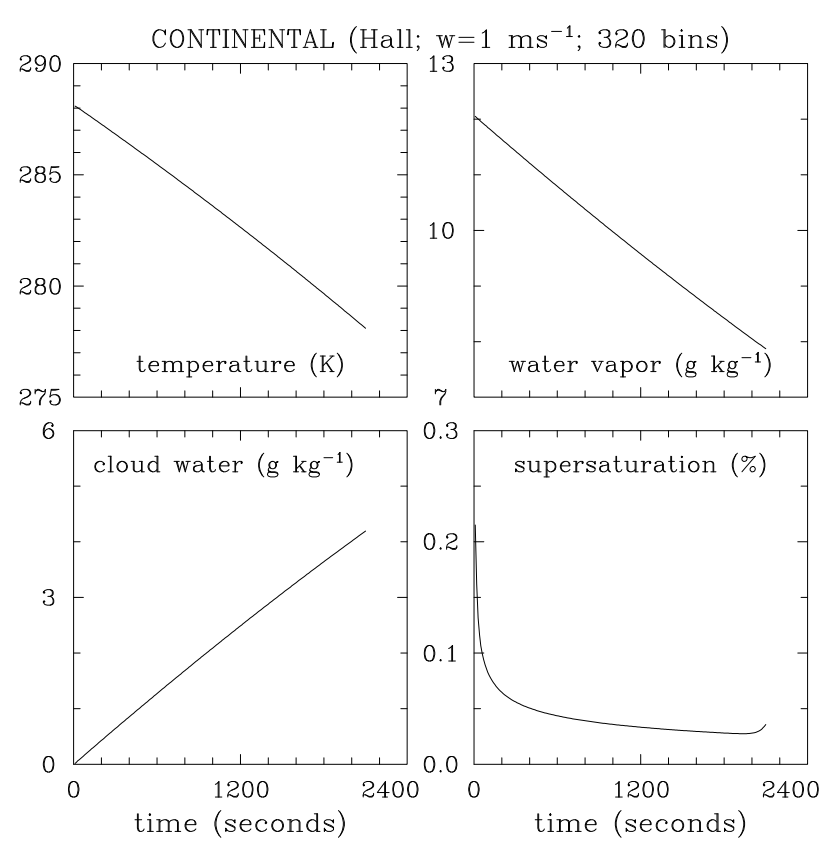

Fig. 3. Time evolution of the temperature, water vapor and cloud water mixing ratios, and supersaturation for the CONTINENTAL case simulation with $w=1 \mathrm{~ms}^{-1}$, Hall gravitational kernel, and $\mathcal{N}=320$.

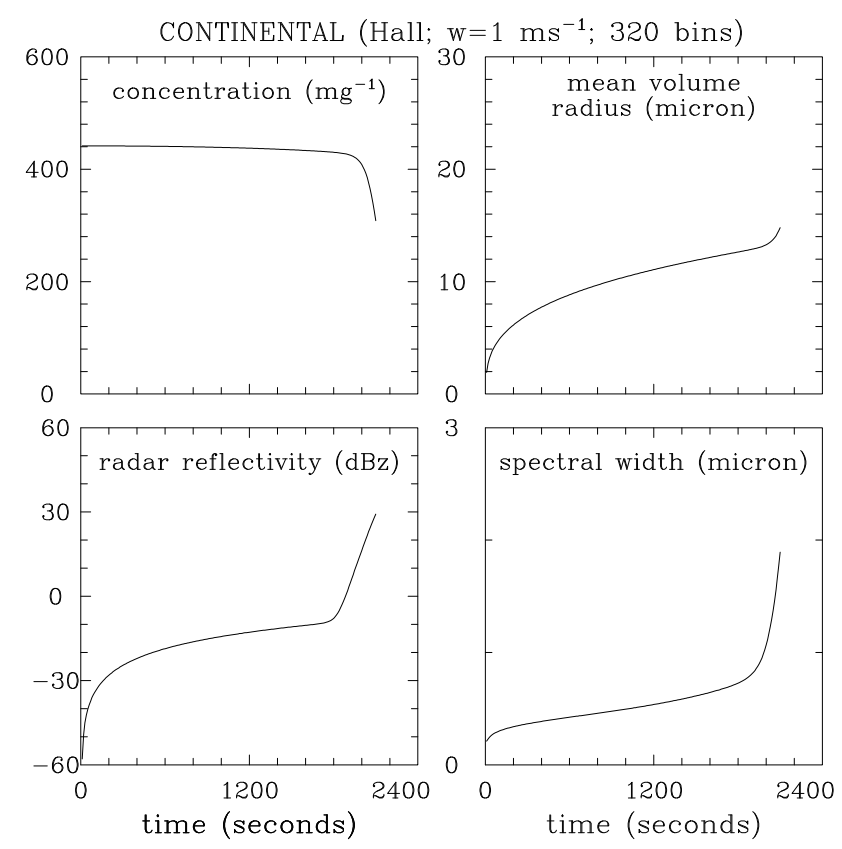

Fig. 4. Time evolution of the drop concentration, mean volume radius, radar reflectivity, and spectral width for the same simulation as Fig. 2 (CONTINENTAL case with $w=1 \mathrm{~m} \mathrm{~s}^{-1}$, Hall gravitational kernel, and $\mathcal{N}=320)$. mixing ratio, and supersaturation. As the parcel rises, the parcel temperature gradually decreases (the rate corresponds to about $5 \mathrm{~K} \mathrm{~km}^{-1}$, the moist adiabatic lapse rate at these temperatures), the water vapor decreases and the condensed water increases. The total water, the sum of the water vapor and cloud water, does not change. The supersaturation sharply increases in the initial few seconds (not captured by the temporal resolution of the plot) and then gradually decreases throughout the most of the simulation. The rapid increase of the supersaturation early in the simulation corresponds to the activation phase, when both the droplet concentration and supersaturation increase until the supersaturation levels off and the activation is completed. The increase of the supersaturation toward the end of the simulation is due to reduction of the droplet concentration, when drizzle and raindrops rapidly remove cloud droplets (the evolution of the supersaturation depends on the parcel vertical velocity and on the phase relaxation time scale, the latter inversely proportional to the product of the droplet concentration and their mean radius, see for instance Clark and Hall, 1979; Eq. 2.22).

The decrease of the drop concentration toward the end of the simulation is illustrated in Fig. 4 which shows evolution of the drop concentration, mean volume radius (the third moment of the distribution), radar reflectivity (the sixth moment), and spectral width (the standard deviation of the distribution). As the figure shows, the concentration of activated droplets is around $420(\mathrm{mg})^{-1}$. The concentration decreases gently throughout most of the simulation and then rapidly in the final few minutes. Arguably, the former is due the autoconversion phase of the collisional growth, when collisions between droplets of similar sizes widen the spectrum, whereas the latter corresponds to the accretion phase of the collisional growth, when drizzle drops efficiently collect cloud droplets (Berry and Reinhardt, 1974a). The mean volume radius increases gradually throughout the most of the simulation and quite rapidly near the end, again consistent with the autoconversion and accretion phases of the rain development. The radar reflectivity increases from initial values below $-60 \mathrm{dBz}$ to values close to $-10 \mathrm{dBz}$ quite gradually and then rapidly. The transition from gradual to rapid increase of the radar reflectivity will be used as one of the metrics to identify to onset of precipitation, as discussed later in the paper. The spectral width of the drop spectrum is quite small, around $0.5 \mu \mathrm{m}$, throughout the most of the simulation. The width starts to increas rapidly towards the end of the simulation, arguably due to development of drizzle and rain. Overall, it takes about $35 \mathrm{~min}$ and $2.2 \mathrm{~km}$ of the vertical displacement to develop radar reflectivity of $30 \mathrm{dBz}$.

Figures 5 and 6 are for the MARITIME case. Overall, the evolution of various quantities is similar to the CONTINENTAL case, but there are important differences. First, the supersaturation peak at the activation phase is higher and the concentration of nucleated droplets is lower [around $\left.90(\mathrm{mg})^{-1}\right]$. The mean volume radius increases faster since there are fewer droplets, and the rapid increase of the radar 


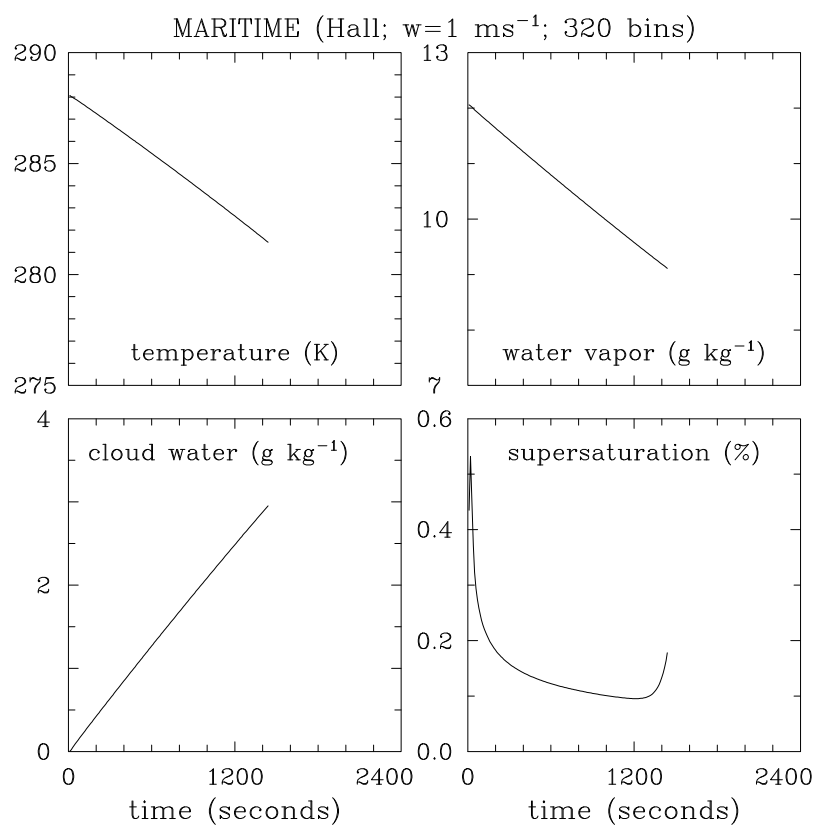

Fig. 5. As Fig. 3, but for the corresponding MARITIME case.

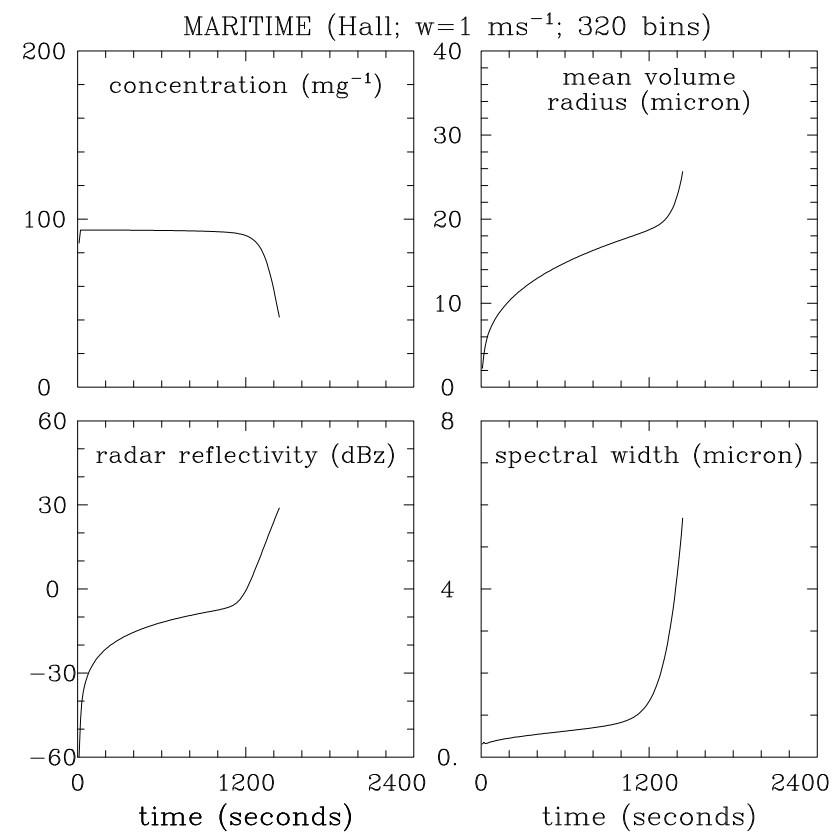

Fig. 6. As Fig. 4, but for the corresponding MARITIME case.

reflectivity (starting again at around $-10 \mathrm{dBz}$ ) happens earlier in the simulation. The mean volume radius at this transition is larger than for the CONTINENTAL case (around 18 versus around $12 \mu \mathrm{m}$ ) and the radar reflectivity of $30 \mathrm{dBz}$ is reached at a lower altitude, around $1.4 \mathrm{~km}$, in about $23 \mathrm{~min}$ of the parcel rise. These differences are consistent with numerous observational and modeling studies of cloud processes in continental and maritime environments.

Figures 7 and 8 show the evolution of the mass density distribution (derived from the drop spectra as $\phi^{(i)} q_{i}^{(0)} \Delta r^{(i)} / \log \frac{r^{(i+1 / 2)}}{r^{(i-1 / 2)}}$ for a display on the log-log plot) for CONTINENTAL and MARITIME conditions, respectively, and again for the Hall gravitational kernel and 320-bin simulations. The spectra are shown at times corresponding to radar reflectivities of $-20,-10,0,10$, and $20 \mathrm{dBz}$. Except for the timing and smaller droplet sizes grown by diffusion of water vapor, the evolutions are similar. At $-20 \mathrm{dBz}$ (after about 9 and 4 min for CONTINENTAL and MARITIME cases) the spectra are relatively narrow, centered at about 8 and $10 \mu \mathrm{m}$ for both cases. The spectra become significantly wider at $-10 \mathrm{dBz}$ (28 and $13 \mathrm{~min}$, respectively) due to effects of droplet collisions. This is the autoconversion phase of the collisional growth (Berry and Reinhardt, 1974a). At times corresponding to subsequent radar reflectivities $(0,10$, and $20 \mathrm{dBz}$ ), the drizzle drops (i.e., drops larger than about $100 \mu \mathrm{m})$ appear in appreciable numbers and the concentration of diffusionally-grown droplets begins to decrease (this is more apparent in drop concentration panels of Figs. 4 and $6)$. This stage corresponds to the accretion phase of the collisional growth (Berry and Reinhardt, 1974a). The peak in the drizzle/raindrop part of the spectrum, developed somewhere between times corresponding to -10 and $0 \mathrm{dBz}$, begins to shift towards larger sizes, and a clear minimum separating cloud droplets and drizzle/rain drops is maintained between 30 and $40 \mu \mathrm{m}$.

Figures 9 and 10, in a format similar to Fig. 13 in XWG08, show evolution of the drop growth rate separated into condensational and collisional components, for CONTINENTAL and MARITIME conditions, respectively, and for the same simulations as Figs. 3 to 8. The condensational growth rate illustrates the growth of drops by diffusion of water vapor, with a gradual shift of the drop spectrum towards larger sizes with a tightly packed negative-positive pattern representing advection of the spectral density function towards larger drop sizes. For the collisional growth rate, deviations from a flat zero line during the autoconversion phase (say, around $-10 \mathrm{dBz}$; cf. Figs. 7 and 8 ) are hardly visible in the figures. Comparable growth rates for diffusional and collisional contributions occur only during the accretion phase of collisional growth, when reflectivities exceed about $0 \mathrm{dBz}$.

All simulations performed in this study demonstrate features highlighted above. To facilitate comparison between various simulations, model results were compiled into several tables to document essential differences in the simulations. Some of the differences are due to numerical aspects and some are due to physical processes. The discussion below aims at separating one from the other. 
Table 2. Selected model results at $-30 \mathrm{dBz}$ for MARITIME and CONTINENTAL cases, various grid resolutions, and Hall gravitational kernel. The columns show grid resolution $\mathcal{N}$, time elapsed $t$ and height $h$ of $-30 \mathrm{dBz}$ level, drop concentration $N$, liquid water content $q_{c}$, mean volume radius $r_{v}$, standard deviation of the drop spectrum $\sigma$, and supersaturation $S$.

\begin{tabular}{|c|c|c|c|c|c|c|c|}
\hline Simulation & $\begin{array}{c}t \\
{[\mathrm{~s}]}\end{array}$ & $\begin{array}{c}h \\
{[\mathrm{~m}]}\end{array}$ & $\begin{array}{c}N \\
{\left[(\mathrm{mg})^{-1}\right]}\end{array}$ & $\begin{array}{c}q_{c} \\
{\left[\mathrm{~g} \mathrm{~kg}^{-1}\right]}\end{array}$ & $\begin{array}{c}r_{v} \\
{[\mu \mathrm{m}]}\end{array}$ & $\begin{array}{c}\sigma \\
{[\mu \mathrm{m}]}\end{array}$ & $\begin{array}{c}\mathrm{S} \\
{[\%]}\end{array}$ \\
\hline \multicolumn{8}{|c|}{ MARITIME: } \\
\hline $\mathcal{N}=40$ & 70 . & 70 . & 90. & 0.14 & 7.2 & 1.6 & 0.27 \\
\hline $\mathcal{N}=69$ & 80. & 80 & 94. & 0.16 & 7.5 & 0.93 & 0.26 \\
\hline $\mathcal{N}=80$ & 80. & 80. & 93. & 0.16 & 7.5 & 0.97 & 0.26 \\
\hline $\mathcal{N}=120$ & 80. & 80. & 94. & 0.16 & 7.5 & 0.63 & 0.26 \\
\hline $\mathcal{N}=160$ & 80. & 80. & 94. & 0.16 & 7.5 & 0.59 & 0.26 \\
\hline $\mathcal{N}=200$ & 80. & 80. & 94. & 0.16 & 7.5 & 0.44 & 0.26 \\
\hline $\mathcal{N}=300$ & 80. & 80. & 94. & 0.16 & 7.5 & 0.33 & 0.26 \\
\hline $\mathcal{N}=320$ & 80. & 80. & 94. & 0.16 & 7.5 & 0.37 & 0.26 \\
\hline \multicolumn{8}{|c|}{ CONTINENTAL: } \\
\hline $\mathcal{N}=40$ & 110. & 110. & 424. & 0.24 & 5.1 & 1.30 & 0.08 \\
\hline $\mathcal{N}=69$ & 150. & 150. & 415. & 0.32 & 5.7 & 0.76 & 0.08 \\
\hline $\mathcal{N}=80$ & 150. & 150. & 479. & 0.32 & 5.4 & 0.85 & 0.07 \\
\hline $\mathcal{N}=120$ & 160. & 160. & 417. & 0.34 & 5.8 & 0.52 & 0.07 \\
\hline $\mathcal{N}=160$ & 160. & 160. & 462. & 0.34 & 5.6 & 0.53 & 0.07 \\
\hline $\mathcal{N}=200$ & 160. & 160. & 418. & 0.34 & 5.8 & 0.36 & 0.07 \\
\hline $\mathcal{N}=300$ & 170. & 170. & 438. & 0.36 & 5.8 & 0.27 & 0.07 \\
\hline $\mathcal{N}=320$ & 160. & 160. & 442. & 0.34 & 5.7 & 0.33 & 0.07 \\
\hline
\end{tabular}

As mentioned in the preceding discussion, the differences between CONTINENTAL and MARITIME cases are primarily because of the different concentrations of activated cloud droplets. However, the width of the activation spectrum is also affected by the number of bins applied in the finite difference algorithm. This is illustrated in Table 2, which shows selected model results at the time when the radar reflectivity factor is at $-30 \mathrm{dBz}$ (i.e., soon after the activation of cloud droplets is completed) for $w=1 \mathrm{~m} \mathrm{~s}^{-1}$ CONTINENTAL and MARITIME cases, and applying the Hall collection kernel and various grids, with number of bins from 40 to 320 (cf. Table 1). Since the form of the collection kernel is irrelevant for the activation of cloud droplets, results for all other kernels are virtually the same and thus are omitted. The table shows that the $-30 \mathrm{dBz}$ is reached at height of about 80/160 $\mathrm{m}$ for the MARITIME/CONTINENTAL case. The number of activated droplets shows small dependence on the number of bins in the MARITIME case, but more significant in the CONTINENTAL case. The liquid water mixing ratios, droplet mean volume radii, and supersaturations vary consistently between CONTINENTAL and MARITIME conditions and they weakly depend on the numerical grid applied. However, the width of the droplet spectrum, $\sigma$, decreases significantly when the number of bins $\mathcal{N}$ increases, from values close to $1 \mu \mathrm{m}$ for low resolution to around $0.3 \mu \mathrm{m}$ for the highest ${ }^{4}$. The dependence of $\sigma$ on $\mathcal{N}$ is a combination of

\footnotetext{
${ }^{4}$ Differences between simulations using 300 and 320 bins seem
}

two effects: (i) the dependence of the width of the droplet spectrum at the end of activation on $\mathcal{N}$, and (ii) the widening of the spectrum due to small $\mathcal{N}$ for subsequent diffusional growth (i.e., before the reflectivity reaches $-30 \mathrm{dBz}$ ). By comparing the width of the droplet spectrum at the maximum supersaturation to the data shown in Table 2, it is concluded that both effects contribute to the width at $-30 \mathrm{dBz}$ (not shown). As one might expect, this aspect has significant effect on the development of drizzle and rain as documented in the following discussion.

Tables 3 and 4 present selected results at the time when the radar reflectivity factor is $20 \mathrm{dBz}$ (i.e., when the precipitation-size drops are already present in the parcel, see Figs. 7 and 8), again for $w=1 \mathrm{~m} \mathrm{~s}^{-1}$ MARITIME and CONTINENTAL cases applying various collection kernels (Hall, Long, A100, and A400). In both tables, the development of rain is faster for the Long kernel than for the Hall kernel, and it is the fastest for the A400 kernel. For a given kernel, the development of rain is the fastest with the lowest number of bins. For instance, for the MARITIME/CONTINENTAL cases using Hall kernel, the $20 \mathrm{dBz}$ is reached after $1100 / 1530 \mathrm{~s}$ for $\mathcal{N}=40$ and after $1370 / 2030 \mathrm{~s}$ for $\mathcal{N}=320$, whereas for the A400 kernel corresponding numbers are $880 / 1210$ and 990/1460. The dependence of

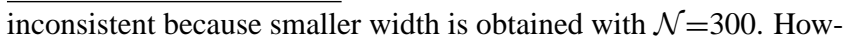
ever, as illustrated in Fig. 1 , the $\mathcal{N}=300$ case features more bins in the diffusional growth range. 
Table 3. Selected model results at $20 \mathrm{dBz}$ for MARITIME case with various kernels and grid resolutions. The data as in Table 2. The symbol (a) in the last column identifies simulations with additional activation of cloud droplets toward the end of the simulation.

\begin{tabular}{|c|c|c|c|c|c|c|c|}
\hline Simulation & $\begin{array}{c}t \\
{[\mathrm{~s}]}\end{array}$ & $\begin{array}{c}h \\
{[\mathrm{~m}]}\end{array}$ & $\begin{array}{c}N \\
{\left[(\mathrm{mg})^{-1}\right]}\end{array}$ & $\begin{array}{c}q_{c} \\
{\left[\mathrm{~g} \mathrm{~kg}^{-1}\right]}\end{array}$ & $\begin{array}{c}r_{v} \\
{[\mu \mathrm{m}]}\end{array}$ & $\begin{array}{c}\sigma \\
{[\mu \mathrm{m}]}\end{array}$ & $\begin{array}{c}\mathrm{S} \\
{[\%]}\end{array}$ \\
\hline \multicolumn{8}{|l|}{ Hall: } \\
\hline $\mathcal{N}=40$ & 1100. & 1100. & 26. & 2.3 & 28. & 10. & 0.27 \\
\hline $\mathcal{N}=69$ & 1200. & 1200. & 35. & 2.4 & 26. & 7.9 & 0.22 \\
\hline $\mathcal{N}=80$ & 1230. & 1230. & 37. & 2.5 & 25. & 7.5 & 0.21 \\
\hline $\mathcal{N}=120$ & 1290. & 1290. & 46. & 2.7 & 24. & 6.1 & 0.17 \\
\hline $\mathcal{N}=160$ & 1330. & 1330. & 53. & 2.7 & 23. & 5.2 & 0.15 \\
\hline $\mathcal{N}=200$ & 1340. & 1340. & 60. & 2.8 & 22. & 4.4 & 0.13 \\
\hline $\mathcal{N}=300$ & 1360. & 1360. & 69. & 2.8 & 21. & 3.5 & 0.12 \\
\hline $\mathcal{N}=320$ & 1370. & 1370. & 69. & 2.8 & 21. & 3.5 & 0.12 \\
\hline \multicolumn{8}{|l|}{ Long: } \\
\hline $\mathcal{N}=40$ & 1060. & 1060. & 25. & 2.2 & 27. & 9.4 & 0.28 \\
\hline $\mathcal{N}=69$ & 1160. & 1160. & 34. & 2.4 & 26. & 7.7 & 0.23 \\
\hline $\mathcal{N}=80$ & 1180. & 1180. & 37. & 2.4 & 25. & 7.1 & 0.21 \\
\hline $\mathcal{N}=120$ & 1240. & 1240. & 41. & 2.6 & 25. & 6.6 & 0.19 \\
\hline $\mathcal{N}=160$ & 1260. & 1260. & 49. & 2.6 & 23. & 5.5 & 0.16 \\
\hline $\mathcal{N}=200$ & 1270. & 1270. & 54. & 2.6 & 23. & 4.9 & 0.14 \\
\hline $\mathcal{N}=300$ & 1290. & 1290. & 61. & 2.7 & 22. & 4.2 & 0.13 \\
\hline $\mathcal{N}=320$ & 1300. & 1300. & 60. & 2.7 & 22. & 4.2 & 0.13 \\
\hline \multicolumn{8}{|l|}{ A100: } \\
\hline $\mathcal{N}=40$ & 950. & 950. & 17. & 2.0 & 30. & 11. & $0.41(a)$ \\
\hline $\mathcal{N}=69$ & 1030. & 1030. & 23. & 2.1 & 28. & 9.4 & $0.33(\mathrm{a})$ \\
\hline $\mathcal{N}=80$ & 1040. & 1040. & 25. & 2.2 & 27. & 9.0 & $0.31(\mathrm{a})$ \\
\hline $\mathcal{N}=120$ & 1080. & 1080. & 30. & 2.2 & 26. & 8.0 & $0.26(a)$ \\
\hline $\mathcal{N}=160$ & 1100. & 1100. & 31. & 2.3 & 26. & 7.6 & $0.25(\mathrm{a})$ \\
\hline $\mathcal{N}=200$ & 1100. & 1100. & 32. & 2.3 & 26. & 7.3 & 0.24 \\
\hline $\mathcal{N}=300$ & 1110. & 1110. & 36. & 2.3 & 25. & 6.7 & 0.22 \\
\hline $\mathcal{N}=320$ & 1120. & 1120. & 34. & 2.3 & 25. & 7.1 & 0.23 \\
\hline \multicolumn{8}{|l|}{ A400: } \\
\hline $\mathcal{N}=40$ & 880. & 880. & 16. & 1.8 & 30 & 14. & $0.56(a)$ \\
\hline $\mathcal{N}=69$ & 940. & 940. & 14. & 2.0 & 32. & 12. & 0.49 (a) \\
\hline $\mathcal{N}=80$ & 940. & 940. & 14. & 2.0 & 32. & 12. & 0.49 (a) \\
\hline $\mathcal{N}=120$ & 970. & 970. & 14. & 2.0 & 33. & 12. & 0.49 (a) \\
\hline $\mathcal{N}=160$ & 980. & 980. & 14. & 2.0 & 32. & 12. & $0.47(a)$ \\
\hline $\mathcal{N}=200$ & 980. & 980. & 16. & 2.0 & 31. & 11. & $0.44(a)$ \\
\hline $\mathcal{N}=300$ & 980. & 980. & 17. & 2.0 & 31. & 11. & $0.42(a)$ \\
\hline $\mathcal{N}=320$ & 990. & 990. & 15. & 2.1 & 32. & 11. & 0.45 (a) \\
\hline
\end{tabular}

the 20-dBz-timing on the collection kernel is clearly physical (e.g., see XWG08), but on $\mathcal{N}$ is numerical. Arguably, faster development of rain in simulations with low $\mathcal{N}$ is a combination of (i) a wider droplet spectrum grown by diffusion of water vapor (resulting from both the increased width of the spectrum at the end of activation and the numerical broadening during diffusional growth due to the small number of bins), and (ii) broadening of the spectrum during the growth by collision-coalescence. One can argue that the results with large number of bins (say, 200, 300, and 320) can be viewed as not far from converged solutions, that is, solutions obtained with sufficiently large number of bins. Although that might be approximately true for the timing of the precipitation development, it is not true for the droplet activation as shown in Table 2. In the MARITIME case, rapid development of precipitation and washout of small cloud droplets near the end of the simulation results in an increase of the supersaturation above values encountered at the cloud base. In such cases, additional activation of cloud droplets has to take place in the parcel. All simulation with A400 kernel 
Table 4. As Table 3, but for the CONTINENTAL case.

\begin{tabular}{|c|c|c|c|c|c|c|c|}
\hline Simulation & $\begin{array}{c}t \\
{[\mathrm{~s}]}\end{array}$ & $\begin{array}{c}h \\
{[\mathrm{~m}]}\end{array}$ & $\begin{array}{c}N \\
{\left[(\mathrm{mg})^{-1}\right]}\end{array}$ & $\begin{array}{c}q_{c} \\
{\left[\mathrm{~g} \mathrm{~kg}^{-1}\right]}\end{array}$ & $\begin{array}{c}r_{v} \\
{[\mu \mathrm{m}]}\end{array}$ & $\begin{array}{c}\sigma \\
{[\mu \mathrm{m}]}\end{array}$ & $\begin{array}{c}\mathrm{S} \\
{[\%]}\end{array}$ \\
\hline \multicolumn{8}{|l|}{ Hall: } \\
\hline $\mathcal{N}=40$ & 1530. & 1530. & 251. & 3.1 & 14. & 3.6 & 0.05 \\
\hline $\mathcal{N}=69$ & 1730. & 1730. & 294. & 3.5 & 14. & 2.7 & 0.04 \\
\hline $\mathcal{N}=80$ & 1820. & 1820. & 357. & 3.7 & 13. & 2.4 & 0.03 \\
\hline $\mathcal{N}=120$ & 1880. & 1880. & 327. & 3.8 & 14. & 2.1 & 0.04 \\
\hline $\mathcal{N}=160$ & 1970. & 1970. & 384. & 4.0 & 14. & 1.7 & 0.03 \\
\hline $\mathcal{N}=200$ & 1960. & 1960. & 353. & 3.9 & 14. & 1.6 & 0.03 \\
\hline $\mathcal{N}=300$ & 2020. & 2020. & 391. & 4.1 & 14. & 1.2 & 0.03 \\
\hline $\mathcal{N}=320$ & 2030. & 2030 . & 393. & 4.1 & 14. & 1.2 & 0.03 \\
\hline \multicolumn{8}{|l|}{ Long: } \\
\hline $\mathcal{N}=40$ & 1380. & 1380. & 279. & 2.8 & 13. & 3.3 & 0.05 \\
\hline $\mathcal{N}=69$ & 1610. & 1610. & 311. & 3.3 & 14. & 2.4 & 0.04 \\
\hline $\mathcal{N}=80$ & 1690. & 1690. & 376. & 3.4 & 13. & 2.1 & 0.03 \\
\hline $\mathcal{N}=120$ & 1760. & 1760. & 334. & 3.6 & 14. & 2.0 & 0.04 \\
\hline $\mathcal{N}=160$ & 1850. & 1850. & 373. & 3.7 & 13. & 1.8 & 0.03 \\
\hline $\mathcal{N}=200$ & 1840. & 1840. & 350. & 3.7 & 14. & 1.6 & 0.03 \\
\hline $\mathcal{N}=300$ & 1890. & 1890. & 393. & 3.8 & 13. & 1.2 & 0.03 \\
\hline $\mathcal{N}=320$ & 1910. & 1910. & 386. & 3.8 & 13. & 1.3 & 0.03 \\
\hline \multicolumn{8}{|l|}{ A100: } \\
\hline $\mathcal{N}=40$ & 1320. & 1320. & 189. & 2.7 & 15. & 4.1 & 0.07 \\
\hline $\mathcal{N}=69$ & 1500. & 1500. & 228. & 3.1 & 15. & 3.3 & 0.05 \\
\hline $\mathcal{N}=80$ & 1570. & 1570. & 272. & 3.2 & 14. & 3.0 & 0.05 \\
\hline $\mathcal{N}=120$ & 1600. & 1600. & 233. & 3.2 & 15. & 3.0 & 0.05 \\
\hline $\mathcal{N}=160$ & 1670. & 1670. & 274. & 3.4 & 14. & 2.6 & 0.04 \\
\hline $\mathcal{N}=200$ & 1640. & 1640. & 259. & 3.3 & 15. & 2.5 & 0.05 \\
\hline $\mathcal{N}=300$ & 1670. & 1670. & 289. & 3.4 & 14. & 2.2 & 0.04 \\
\hline $\mathcal{N}=320$ & 1690. & 1690. & 277. & 3.4 & 14. & 2.3 & 0.04 \\
\hline \multicolumn{8}{|l|}{ A400: } \\
\hline $\mathcal{N}=40$ & 1210. & 1210. & 127. & 2.5 & 17. & 5.1 & 0.10 \\
\hline $\mathcal{N}=69$ & 1360. & 1360. & 134. & 2.8 & 17. & 4.7 & 0.09 \\
\hline $\mathcal{N}=80$ & 1410. & 1410. & 157. & 2.9 & 16. & 4.4 & 0.08 \\
\hline $\mathcal{N}=120$ & 1410. & 1410. & 127. & 3.0 & 18. & 4.7 & 0.09 \\
\hline $\mathcal{N}=160$ & 1460. & 1460. & 159. & 3.0 & 17. & 4.2 & 0.08 \\
\hline $\mathcal{N}=200$ & 1430. & 1430. & 149. & 2.9 & 17. & 4.1 & 0.08 \\
\hline $\mathcal{N}=300$ & 1450. & 1450. & 170. & 3.0 & 16. & 3.7 & 0.07 \\
\hline $\mathcal{N}=320$ & 1460. & 1460. & 170. & 3.0 & 16. & 3.8 & 0.07 \\
\hline
\end{tabular}

and more than a half in A100 kernel experience this in-cloud activation as marked in Table 3 .

\subsection{Rain initiation and speedup due to turbulent colli- sions}

The transition from slow to rapid increase of the radar reflectivity between -10 and $0 \mathrm{dBz}$ (see Figs. 4, 6, 7 and 8) corresponds to the development of a new peak in the drop size distributions for drops with radius around $100 \mu \mathrm{m}$ (i.e., drizzle). This specific time will be referred to as the radar reflectivity transition time and it will be used as one of the two measures to precisely define and compare the time of warm rain initiation in various model simulations. Mathematically, the radar reflectivity transition time can be defined as the time of the maximum second derivative (i.e., the maximum curvature) of the radar reflectivity as a function of time for the reflectivity range of -10 to $0 \mathrm{dBz}$. The motivation for using radar reflectivity as a measure of rain initiation time is the possibility of a direct comparison between model results and field observations. 
Table 5. Selected model results at the radar reflectivity transition time for MARITIME and CONTINENTAL cases, various grid resolutions and collision kernels. The columns show grid resolution $\mathcal{N}$, time elapsed $t$ and height reached $h$, radar reflectivity $Z$, drop concentration $N$, liquid water content $q_{c}$, mean volume radius $r_{v}$, and standard deviation of the drop spectrum $\sigma$.

\begin{tabular}{|c|c|c|c|c|c|c|c|}
\hline Simulation & $\begin{array}{c}t \\
{[\mathrm{~s}]}\end{array}$ & $\begin{array}{c}h \\
{[\mathrm{~m}]}\end{array}$ & $\begin{array}{c}Z \\
{[\mathrm{dBz}]}\end{array}$ & $\begin{array}{c}N \\
{\left[(\mathrm{mg})^{-1}\right]}\end{array}$ & $\begin{array}{c}q_{c} \\
{\left[\mathrm{~g} \mathrm{~kg}^{-1}\right]}\end{array}$ & $\begin{array}{c}r_{v} \\
{[\mu \mathrm{m}]}\end{array}$ & $\begin{array}{c}\sigma \\
{[\mu \mathrm{m}]}\end{array}$ \\
\hline \multicolumn{8}{|c|}{ MARITIME, Hall: } \\
\hline $\mathcal{N}=40$ & 860. & 860. & -1.3 & 76. & 1.80 & 17.8 & 3.9 \\
\hline $\mathcal{N}=69$ & 970. & 970. & -2.1 & 84. & 2.03 & 17.9 & 2.9 \\
\hline $\mathcal{N}=80$ & 1000. & 1000. & -2.3 & 84. & 2.09 & 18.1 & 2.6 \\
\hline $\mathcal{N}=120$ & 1070. & 1070. & -2.6 & 88. & 2.23 & 18.2 & 2.1 \\
\hline $\mathcal{N}=160$ & 1100. & 1100. & -2.9 & 89. & 2.31 & 18.4 & 1.7 \\
\hline $\mathcal{N}=200$ & 1130. & 1130. & -3.0 & 90. & 2.35 & 18.4 & 1.5 \\
\hline $\mathcal{N}=300$ & 1170. & 1170. & -2.4 & 91. & 2.43 & 18.5 & 1.3 \\
\hline $\mathcal{N}=320$ & 1170. & 1170. & -3.0 & 91. & 2.43 & 18.5 & 1.2 \\
\hline \multicolumn{8}{|c|}{ MARITIME, A100: } \\
\hline $\mathcal{N}=69$ & 780. & 780. & -4.9 & 85. & 1.64 & 16.7 & 2.6 \\
\hline $\mathcal{N}=160$ & 880. & 880. & -4.6 & 83. & 1.84 & 17.5 & 2.0 \\
\hline $\mathcal{N}=320$ & 910. & 910. & -4.3 & 82. & 1.91 & 17.7 & 1.8 \\
\hline \multicolumn{8}{|c|}{ MARITIME, A400: } \\
\hline $\mathcal{N}=69$ & 670. & 670. & -6.0 & 80. & 1.41 & 16.2 & 2.7 \\
\hline $\mathcal{N}=160$ & 730. & 730. & -5.7 & 75. & 1.54 & 17.0 & 2.3 \\
\hline $\mathcal{N}=320$ & 740. & 740. & -6.2 & 75. & 1.56 & 17.0 & 2.1 \\
\hline \multicolumn{8}{|c|}{ CONTINENTAL, Hall: } \\
\hline $\mathcal{N}=40$ & 1250. & 1250. & -5.8 & 392. & 2.60 & 11.6 & 2.4 \\
\hline $\mathcal{N}=69$ & 1490. & 1490. & -6.3 & 389. & 3.06 & 12.3 & 1.7 \\
\hline $\mathcal{N}=80$ & 1590. & 1590. & -6.6 & 449. & 3.25 & 12.0 & 1.6 \\
\hline $\mathcal{N}=120$ & 1670. & 1670. & -5.7 & 397. & 3.40 & 12.7 & 1.3 \\
\hline $\mathcal{N}=160$ & 1770. & 1770. & -5.8 & 442. & 3.59 & 12.5 & 1.1 \\
\hline $\mathcal{N}=200$ & 1750. & 1750. & -6.2 & 403. & 3.55 & 12.8 & 0.94 \\
\hline $\mathcal{N}=300$ & 1810. & 1810. & -6.3 & 426. & 3.66 & 12.7 & 0.75 \\
\hline $\mathcal{N}=320$ & 1830. & 1830. & -5.9 & 429. & 3.70 & 12.7 & 0.75 \\
\hline \multicolumn{8}{|c|}{ CONTINENTAL, A100: } \\
\hline $\mathcal{N}=69$ & 1260. & 1260. & -7.4 & 380. & 2.61 & 11.8 & 1.7 \\
\hline $\mathcal{N}=160$ & 1450. & 1450. & -7.6 & 401. & 2.98 & 12.1 & 1.3 \\
\hline $\mathcal{N}=320$ & 1480. & 1480. & -7.3 & 377. & 3.04 & 12.4 & 1.2 \\
\hline \multicolumn{8}{|c|}{ CONTINENTAL, A400: } \\
\hline $\mathcal{N}=69$ & 1100. & 1100. & -8.5 & 351. & 2.29 & 11.6 & 1.8 \\
\hline $\mathcal{N}=160$ & 1230. & 1230. & -8.1 & 356. & 2.55 & 12.0 & 1.6 \\
\hline $\mathcal{N}=320$ & 1250. & 1250. & -7.5 & 331. & 2.59 & 12.3 & 1.6 \\
\hline
\end{tabular}

The rain initiation time can also be defined based on the evolution of the growth rate by collision-coalescence shown at right panels of Figs. 9 and 10. XWG08 proposed to specify the rain initiation time as the time when the autoconversion phase finishes and the accretion phase begins, and formally define the boundary between the two phases by the sudden increase of the drop radius corresponding to the maximum growth rate due to collision-coalescence (see Figs. 13 and 14 in XWG08 and the accompanying discussion). This time will be referred to as the autoconversion-accretion transition time. In the model data for low resolution ( (mall $\mathcal{N}$ ), the increase of the drop radius corresponding to the maximum collisional growth rate is rather gradual, so the additional condition used to define the autoconversion-accretion transition time is that the radius of the maximum has to be larger than $60 \mu \mathrm{m}$.

Table 5 compiles various quantities predicted by the parcel model at the radar reflectivity transition time, for MARITIME and CONTINENTAL cases with $1 \mathrm{~m} \mathrm{~s}^{-1}$ updraft and various collection kernels. Results shown in the table are consistent with various features of parcel model results discussed already. For instance, the transition happens earlier in the MARITIME cases when compared to the corresponding CONTINENTAL cases; for a given kernel and the CCN type, rain initiation is the fastest/slowest for grid configurations 
Table 6. As Table 5, but for the autoconversion-accretion transition time.

\begin{tabular}{|c|c|c|c|c|c|c|c|}
\hline Simulation & $\begin{array}{c}t \\
{[\mathrm{~s}]}\end{array}$ & $\begin{array}{c}h \\
{[\mathrm{~m}]}\end{array}$ & $\begin{array}{c}Z \\
{[\mathrm{dBz}]}\end{array}$ & $\begin{array}{c}N \\
{\left[(\mathrm{mg})^{-1}\right]}\end{array}$ & $\begin{array}{c}q_{c} \\
{\left[\mathrm{~g} \mathrm{~kg}^{-1}\right]}\end{array}$ & $\begin{array}{c}r_{v} \\
{[\mu \mathrm{m}]}\end{array}$ & $\begin{array}{c}\sigma \\
{[\mu \mathrm{m}]}\end{array}$ \\
\hline \multicolumn{8}{|c|}{ MARITIME, Hall: } \\
\hline $\mathcal{N}=40$ & 880. & 880. & 0.0 & 74. & 1.84 & 18.1 & 4.1 \\
\hline $\mathcal{N}=69$ & 1000. & 1000. & -0.1 & 81. & 2.09 & 18.3 & 3.2 \\
\hline $\mathcal{N}=80$ & 1000. & 1000. & -2.3 & 84. & 2.09 & 18.1 & 2.6 \\
\hline $\mathcal{N}=120$ & 1040. & 1040. & -4.3 & 89. & 2.16 & 18.0 & 2.0 \\
\hline $\mathcal{N}=160$ & 1100. & 1100. & -2.9 & 89. & 2.31 & 18.4 & 1.7 \\
\hline $\mathcal{N}=200$ & 1150. & 1150. & -1.5 & 89. & 2.39 & 18.5 & 1.7 \\
\hline $\mathcal{N}=300$ & 1200. & 1200. & 0.3 & 90. & 2.49 & 18.8 & 1.4 \\
\hline $\mathcal{N}=320$ & 1210. & 1210. & 0.4 & 90. & 2.51 & 18.8 & 1.4 \\
\hline \multicolumn{8}{|c|}{ MARITIME, A100: } \\
\hline $\mathcal{N}=69$ & 810. & 780. & -2.6 & 82. & 1.70 & 17.1 & 2.8 \\
\hline $\mathcal{N}=160$ & 880. & 880. & -4.6 & 83. & 1.84 & 17.5 & 2.0 \\
\hline $\mathcal{N}=320$ & 980. & 980. & 3.4 & 75. & 2.05 & 18.7 & 2.7 \\
\hline \multicolumn{8}{|c|}{ MARITIME, A400: } \\
\hline $\mathcal{N}=69$ & 700. & 700. & -3.4 & 76. & 1.48 & 16.7 & 3.0 \\
\hline $\mathcal{N}=160$ & 730. & 730. & -5.7 & 75. & 1.54 & 17.0 & 2.3 \\
\hline $\mathcal{N}=320$ & 800. & 800. & 0.2 & 66. & 1.68 & 18.2 & 3.1 \\
\hline \multicolumn{8}{|c|}{ CONTINENTAL, Hall: } \\
\hline $\mathcal{N}=40$ & 1310. & 1310. & -1.6 & 384. & 2.71 & 11.9 & 2.5 \\
\hline $\mathcal{N}=69$ & 1580. & 1580. & 2.0 & 379. & 3.23 & 12.7 & 1.8 \\
\hline $\mathcal{N}=80$ & 1680. & 1680. & 2.1 & 439. & 3.42 & 12.3 & 1.7 \\
\hline $\mathcal{N}=120$ & 1760. & 1760. & 4.2 & 388. & 3.57 & 13.0 & 1.4 \\
\hline $\mathcal{N}=160$ & 1770. & 1770. & -5.8 & 442. & 3.59 & 12.5 & 1.1 \\
\hline $\mathcal{N}=200$ & 1870. & 1870. & 8.4 & 394. & 3.77 & 13.2 & 1.1 \\
\hline $\mathcal{N}=300$ & 1950. & 1950. & 11.5 & 416. & 3.92 & 13.1 & 0.93 \\
\hline $\mathcal{N}=320$ & 1960. & 1960. & 10.6 & 420. & 3.94 & 13.1 & 0.92 \\
\hline \multicolumn{8}{|c|}{ CONTINENTAL, A100: } \\
\hline $\mathcal{N}=69$ & 1330. & 1330. & -0.5 & 369. & 2.74 & 12.1 & 1.8 \\
\hline $\mathcal{N}=160$ & 1450. & 1450. & -7.6 & 401. & 2.98 & 12.1 & 1.3 \\
\hline $\mathcal{N}=320$ & 1620. & 1620. & 11.2 & 342. & 3.30 & 13.2 & 1.7 \\
\hline \multicolumn{8}{|c|}{ CONTINENTAL, A400: } \\
\hline $\mathcal{N}=69$ & 1160. & 1160. & -2.8 & 335. & 2.41 & 12.0 & 2.0 \\
\hline $\mathcal{N}=160$ & 1230. & 1230. & -8.1 & 356. & 2.55 & 12.0 & 1.6 \\
\hline $\mathcal{N}=320$ & 1330. & 1330. & 2.3 & 304. & 2.74 & 12.9 & 2.0 \\
\hline
\end{tabular}

with small/large $\mathcal{N}$; the mean volume radius at the rain initiation time is around $18 \mu \mathrm{m}$ in MARITIME cases and around $12 \mu \mathrm{m}$ in the CONTINENTAL cases. Another relevant features include (i) only slightly reduced drop concentration compared to the values just after activation (cf. Table 2); (ii) systematically decreasing spectral width $\sigma$ with increasing $\mathcal{N}$ (by a factor larger than 3 between 40 and 320 bins); and (iii) significantly larger $\sigma$ for simulations using A400 kernel compared to corresponding simulations using Hall kernel, especially when using large number of bins (by a factor close to 2 for both MARITIME and CONTINENTAL cases). While (ii) is clearly associated with numerical aspects, (iii) is most likely related to significantly increased A400 collection kernel compared to gravitational kernels.
Table 6 compiles the same quantities as Table 5, but for the autoconversion-accretion transition time. In general, the results are similar to the radar reflectivity transition time, but the corresponding times (and thus heights, radar reflectivities, liquid water mixing ratios, mean volume radii, and spectral widths) are larger. For the Hall gravitational kernel, the difference between corresponding heights in Tables 5 and 6 is 20 to $40 \mathrm{~m}$ for the MARITIME case and 60 to $140 \mathrm{~m}$ for the CONTINENTAL case depending on $\mathcal{N}$. The drop concentration is also slightly reduced. The corresponding radar reflectivity varies significantly for the CONTINENTAL case as a function of $\mathcal{N}$. 


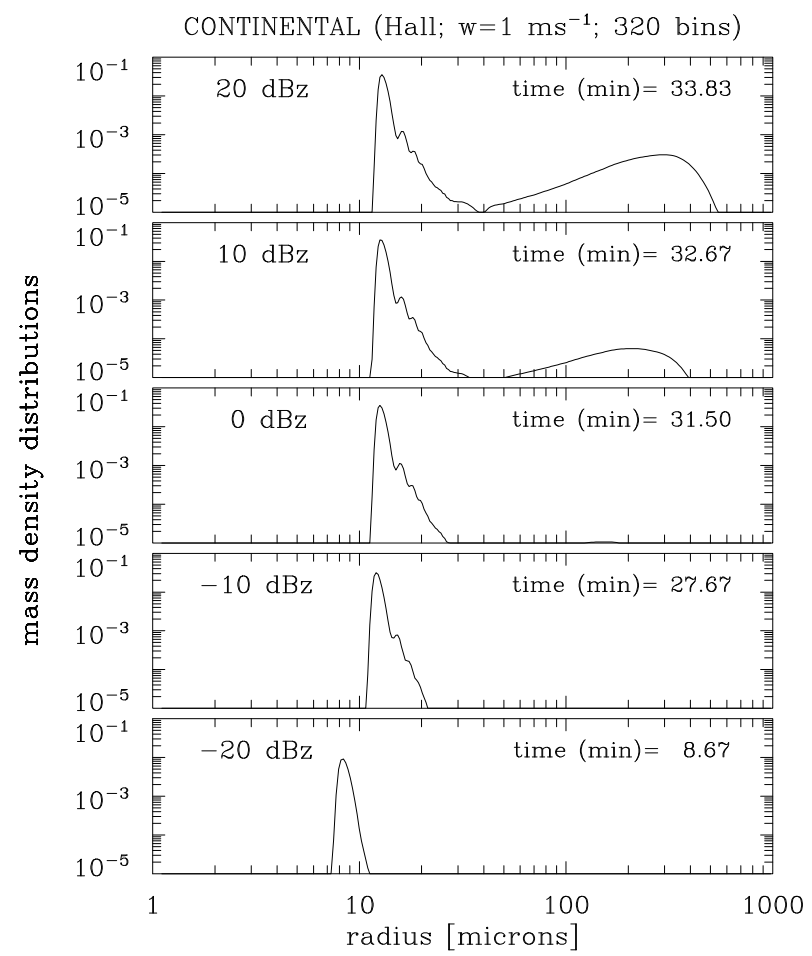

Fig. 7. Mass density distributions for times corresponding to radar reflectivities of $-20,-10,0,10$, and $20 \mathrm{dBz}$ for the CONTINENTAL case with $w=1 \mathrm{~m} \mathrm{~s}^{-1}$, Hall gravitational kernel, and $\mathcal{N}=320$. The time needed to reach the given reflectivity is shown in each panel as well.

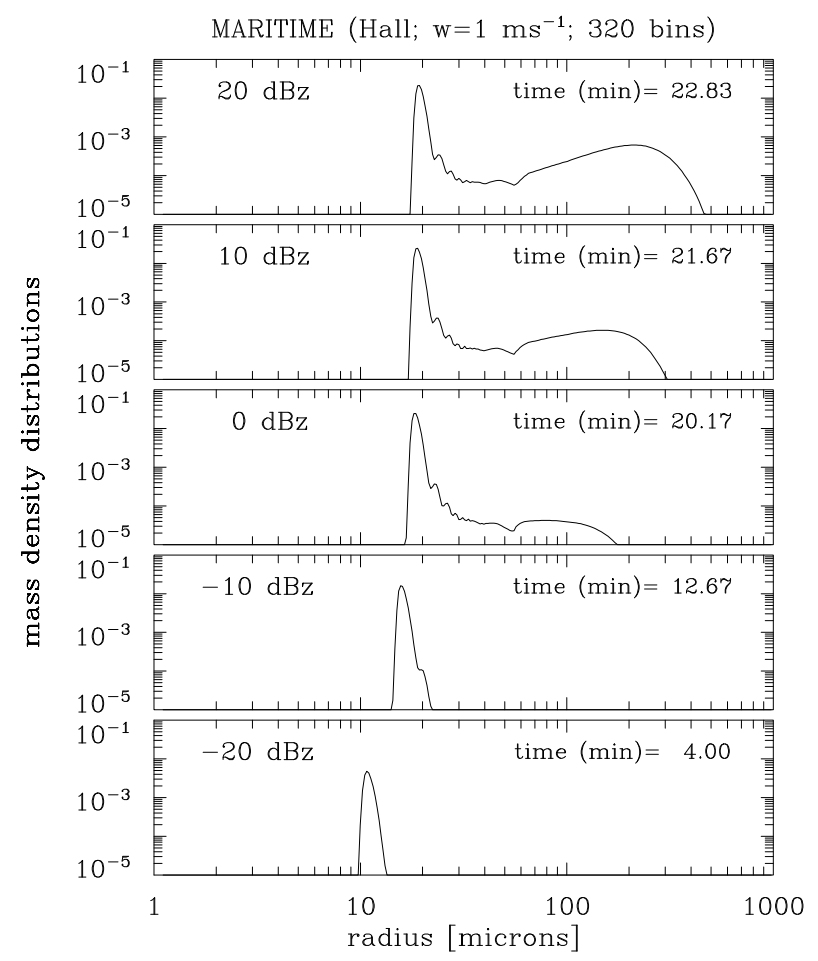

Fig. 8. As Fig. 7, but for the MARITIME case.

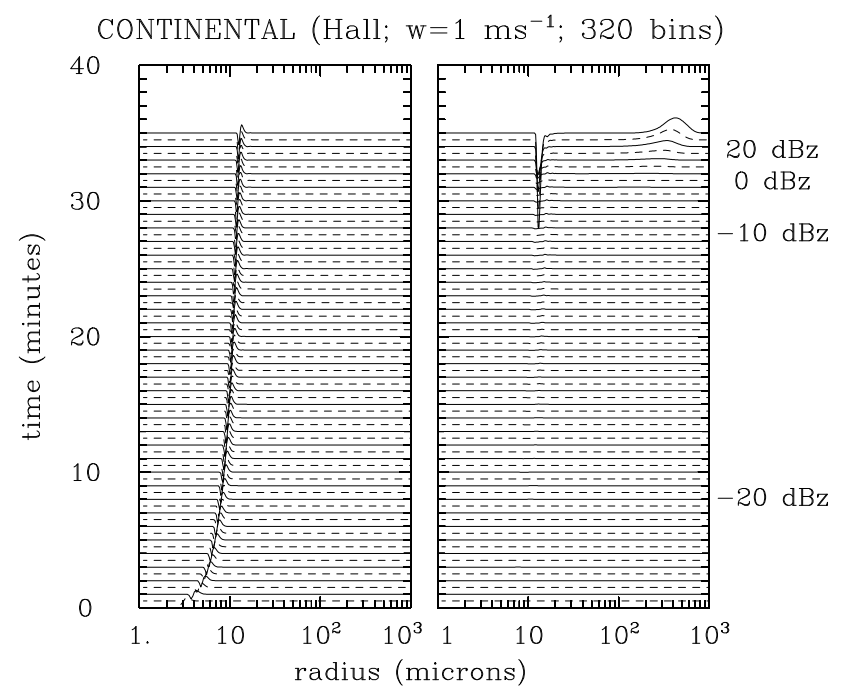

Fig. 9. Evolution of the mass transfer rate $\partial \phi^{(i)} / \partial t q_{i}^{(0)} \Delta r^{(i)} / \log \frac{r^{(i+1 / 2)}}{r^{(i-1 / 2)}}$ as a function of drop radius $r$ separated into condensational growth (left panel) and collisional growth (right panel) for the CONTINENTAL case with $w=1 \mathrm{~m} \mathrm{~s}^{-1}$, Hall gravitational kernel, and $\mathcal{N}=320$. The vertical axis represents time, and the rate at $t=0$ (at the end of the simulation) is shown at the bottom (top) of each panel. Growth rates are plotted every half minute for the entire simulation using solid/dashed lines for full/half minutes. The flat (zero) part of each transfer rate marks the corresponding time. The scale of the mass transfer rate is such that $1 \mathrm{~min}$ of the time scale corresponds to approximately 3.0 and $0.6 \mathrm{mg} \mathrm{kg}^{-1} \mathrm{~s}^{-1}$ of the mass transfer rate for condensational and collisional growth, respectively. Reflectivities at the right-hand-side of the plot mark approximate times a given reflectivity is reached.

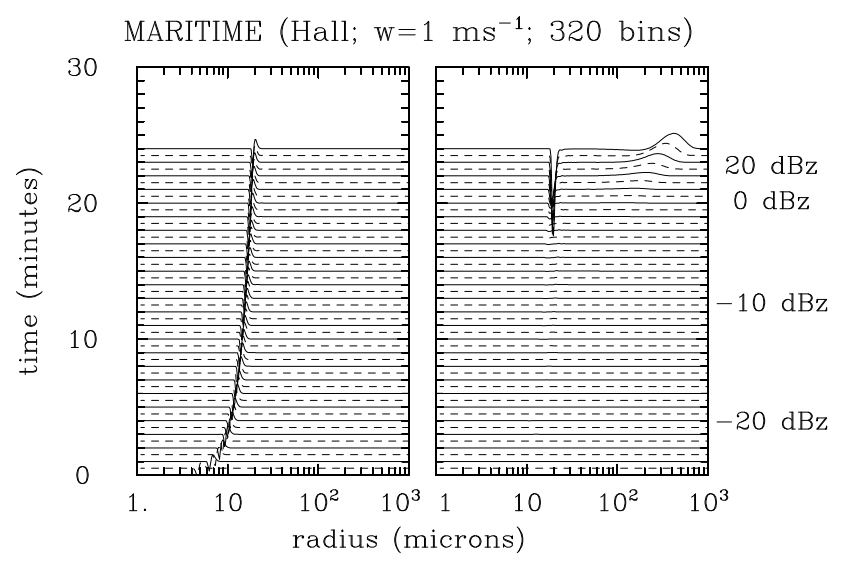

Fig. 10. As Fig. 9, but for the MARITIME case. 


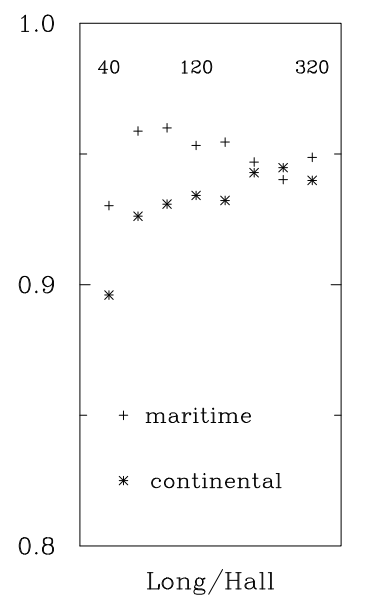

Fig. 11. Ratios of the radar reflectivity transition time using the Long gravitational kernel and the Hall kernel for CONTINENTAL and MARITIME conditions, and for various grid resolutions $\mathcal{N}$. Results for the smallest/largest $\mathcal{N}$ are at the left/right side of the panel.

The rain initiation times defined above (i.e., either the radar reflectivity transition time or the autoconversionaccretion transition time) are used in the analysis of the speedup factor for turbulent collision-coalescence, defined as the ratio of the rain initiation time for the turbulent collection kernel (either A100 or A400) and the corresponding time for the Hall gravitational kernel. Before showing the turbulent speedup factors, however, we first show the corresponding ratio between the radar reflectivity transition times using the Long and Hall kernels. The motivation is that both the Long and Hall kernels represent different approximations for the gravitational kernel. One can argue that the Hall kernel is more accurate (e.g., because of a more accurate formulation of droplet terminal velocity or more up-to-date collision efficiency data), but the differences between results obtained using the two kernels can be used to assess the differences between gravitational and turbulent kernels.

Figure 11 shows the ratio of the radar reflectivity transition time for the Long and Hall kernels, for CONTINENTAL and MARITIME cases, and for simulations with different $\mathcal{N}$. Despite significant differences in the rain initiation times for various $\mathcal{N}$ (factor of almost 1.5 between 40 and 320 in Table 5), the ratio between times for Long and Hall kernels is between 0.90 and 0.95 for most $\mathcal{N}$. As the $\mathcal{N}$ increases, the ratio approaches 0.95 for both CONTINENTAL and MARITIME cases. This value can be compared to the turbulent speedup factors for A100 and A400 kernels shown in Fig. 12 (applying the radar reflectivity transition times) and 13 (applying the autoconversion-accretion transition times). In general, the speedup factors are quite similar using either definition of the transition time. Despite significant dependence of the rain initiation times on $\mathcal{N}$, the turbu-

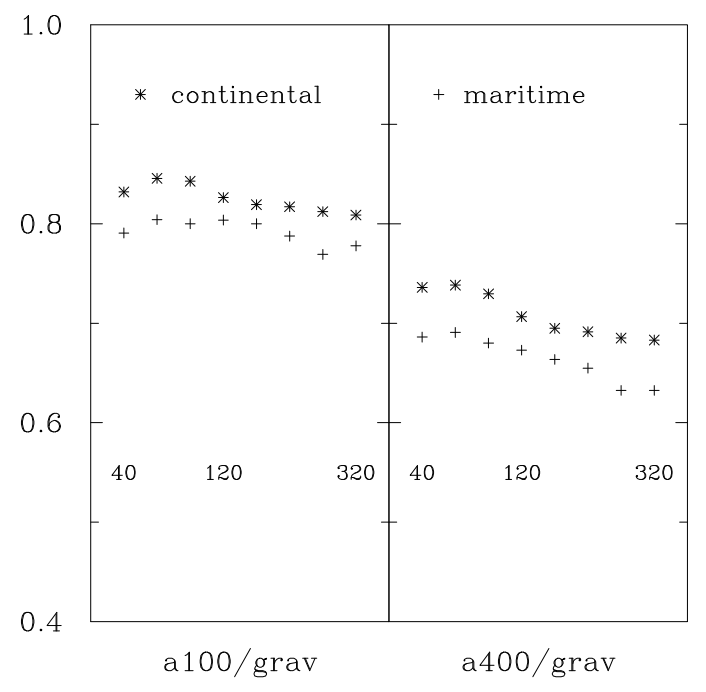

Fig. 12. As Fig. 11, but for the ratios between the radar reflectivity transition times for A100 (left panel) or A400 (right panel) and the Hall gravitational kernel.

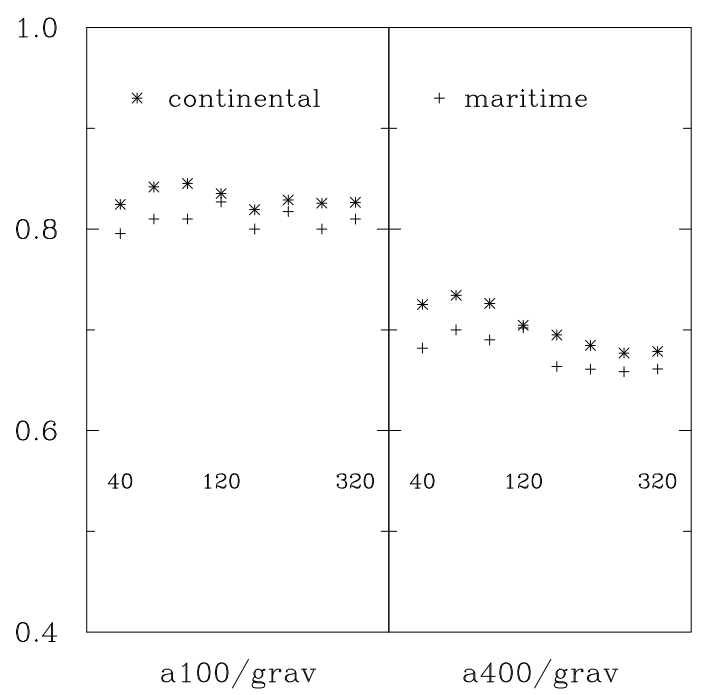

Fig. 13. As Fig. 11, but for the ratios between the autoconversionaccretion transition times for A100 (left panel) or A400 (right panel) and the Hall gravitational kernel.

lent speedup factor only weakly depends on the bin resolution. Overall, the speedup factors seem to decrease slightly with $\mathcal{N}$ and are slightly larger for the CONTINENTAL case. The speedup factors for A100 kernel are around 0.8, and for A400 the factors are between 0.65 and 0.75 .

By comparing Figs. 11, 12, and 13, one can clearly see that turbulent enhancement is significantly larger than the uncertainty associated with the formulation of the gravitational kernel. Moreover, if results for higher $\mathcal{N}$ are considered 
Table 7. Selected model results for different parcel vertical velocities, MARITIME and CONTINENTAL cases, and various grid resolutions and collection kernels. The columns show the vertical velocity $w$, grid resolution $\mathcal{N}$, droplet concentration corresponding to $Z=-30 \mathrm{dBz}$ $N\left(-30 \mathrm{dBz}\right.$, and several quantities at the radar reflectivity transition time (time elapsed $t$ and height reached $h$, liquid water content $q_{c}$, radar reflectivity $Z$, and mean volume radius $r_{v}$ ).

\begin{tabular}{lcccccccc}
\hline kernel and aerosol type & $\begin{array}{c}w \\
{\left[\mathrm{~m} \mathrm{~s}^{-1}\right]}\end{array}$ & $\begin{array}{c}\mathcal{N} \\
{[1]}\end{array}$ & $\begin{array}{c}N(-30 \mathrm{dBz}) \\
{\left[(\mathrm{mg})^{-1}\right]}\end{array}$ & $\begin{array}{c}t \\
{[\mathrm{~s}]}\end{array}$ & $\begin{array}{c}H \\
{[\mathrm{~m}]}\end{array}$ & $\begin{array}{c}q_{c} \\
{\left[\mathrm{~g} \mathrm{~kg}^{-1}\right]}\end{array}$ & $\begin{array}{c}Z \\
{[\mathrm{dBz}]}\end{array}$ & $\begin{array}{c}r_{v} \\
{[\mu \mathrm{m}]}\end{array}$ \\
\hline MARITIME, Hall & 0.2 & 69 & 62. & 2400. & 480. & 1.02 & -6.6 & 16.2 \\
& 0.2 & 160 & 62. & 2750. & 550. & 1.16 & -6.9 & 16.7 \\
& 0.2 & 320 & 62. & 2900. & 580. & 1.23 & -6.5 & 16.9 \\
& 5.0 & 69 & 141. & 396. & 1980. & 3.97 & 2.0 & 19.8 \\
& 5.0 & 160 & 140. & 456. & 2280. & 4.51 & 1.3 & 20.1 \\
& 5.0 & 320 & 141. & 482. & 2410. & 4.74 & 1.2 & 20.3 \\
\hline MARITIME, A100 & 0.2 & 320 & 62. & 2250. & 450. & 0.96 & -9.4 & 16.1 \\
& 5.0 & 320 & 141. & 362. & 1810. & 3.65 & -0.8 & 19.2 \\
\hline MARITIME, A400 & 0.2 & 320 & 62. & 1900. & 380. & 0.81 & -9.1 & 15.8 \\
& 5.0 & 320 & 141. & 296. & 1480. & 3.02 & -1.9 & 18.6 \\
\hline CONTINENTAL, Hall & 0.2 & 69 & 233. & 3500. & 700. & 1.48 & -10.3 & 11.7 \\
& 0.2 & 160 & 260. & 4150. & 830. & 1.75 & -10.2 & 11.9 \\
& 0.2 & 320 & 249. & 4300. & 860. & 1.81 & -9.7 & 12.1 \\
& 5.0 & 69 & 734. & 646. & 3230. & 6.11 & -2.4 & 12.8 \\
& 5.0 & 160 & 724 & 740. & 3700. & 6.82 & -2.4 & 13.3 \\
& 5.0 & 320 & 764. & 790. & 3950. & 7.18 & -2.8 & 13.2 \\
\hline CONTINENTAL, A400 & 0.2 & 320 & 247. & 2950. & 590. & 1.25 & -11.9 & 11.7 \\
& 5.0 & 320 & 764. & 526. & 2630. & 5.13 & -4.5 & 12.8 \\
\hline \multirow{2}{*}{ CONTINENTAL, A100 } & 0.2 & 320 & 248. & 3500. & 700. & 1.48 & -11.0 & 11.9 \\
& 5.0 & 320 & 764. & 632. & 3160. & 6.00 & -3.6 & 13.0 \\
\hline
\end{tabular}

more reliable, the turbulent kernel corresponding to the turbulent dissipation rate of $100 / 400 \mathrm{~cm}^{2} \mathrm{~s}^{-3}$ can reduce the rain initiation time by about $20 \% / 35 \%$ compared to the gravitational case independent whether the radar reflectivity transition time or the autoconversion-accretion transition time is used as the rain initiation time.

\section{Sensitivity simulations}

To ensure that the results discussed above are robust, a set of sensitivity simulations with $w=0.2$ and $5 \mathrm{~m} \mathrm{~s}^{-1}$ was executed. Table 7 presents selected results from these simulations, for both MARITIME and CONTINENTAL cases and for selected number of bins $\mathcal{N}$. The table shows concentration of droplets at $-30 \mathrm{dBz}$ (i.e., shortly after the activation) and several quantities at the radar reflectivity transition time (the time and height of the transition, liquid water mixing ratio and the mean volume radius). As expected, the concentration of droplets is significantly affected by the vertical velocity: for the MARITIME case the concentration changes from 62 to $141(\mathrm{mg})^{-1}$ for $w$ of 0.2 and $5 \mathrm{~m} \mathrm{~s}^{-1}$. This has significant impact on the rain initiation which for $\mathcal{N}=320$ occurs at times 2900 and $482 \mathrm{~s}$ (heights of 580 and $2410 \mathrm{~m}$ ) for MAR-
ITIME cases with the Hall kernel and $w$ of 0.2 and $5 \mathrm{~m} \mathrm{~s}^{-1}$, respectively. The liquid water mixing ratio at the transition increases with the increasing $w$ from about 1.2 to $4.7 \mathrm{~g} \mathrm{~kg}^{-1}$ for this case (reflectivity increases from -7 to $1 \mathrm{dBz}$ ). For the corresponding CONTINENTAL case, the liquid water increases from 1.8 to $7.2 \mathrm{~g} \mathrm{~kg}^{-1}$ and the reflectivity increases from -10 to $-3 \mathrm{dBz}$. The mean volume radius at the time of the transition varies between 16 and $20 \mu \mathrm{m}$ for the MARITIME case and between 12 and $13 \mu \mathrm{m}$ for the CONTINENTAL case. It follows that the rain initiation time is a sensitive function of the parcel vertical velocity, and it is a combination of different concentrations of droplets activated near the cloud base at different $w$ (as documented in Table 7) and different times when the autoconversion phase of the collisional growth starts. As illustrated in Figs. 7 and 8, the autoconversion phase requires cloud droplets to reach radii around $10 \mu \mathrm{m}$ and reflectivities between -20 and $-10 \mathrm{dBz}$.

Despite such a wide range of rain initiation times for various $w$, the turbulent speedup factors are similar. This is illustrated in Fig. 14 which shows the speedup factors applying the radar reflectivity transition times for various $w, \mathcal{N}$ and either A100 or A400 turbulent collection kernels. As the figure shows, the speedup factors are slightly smaller for the MARITIME case and larger vertical velocities. They are between 


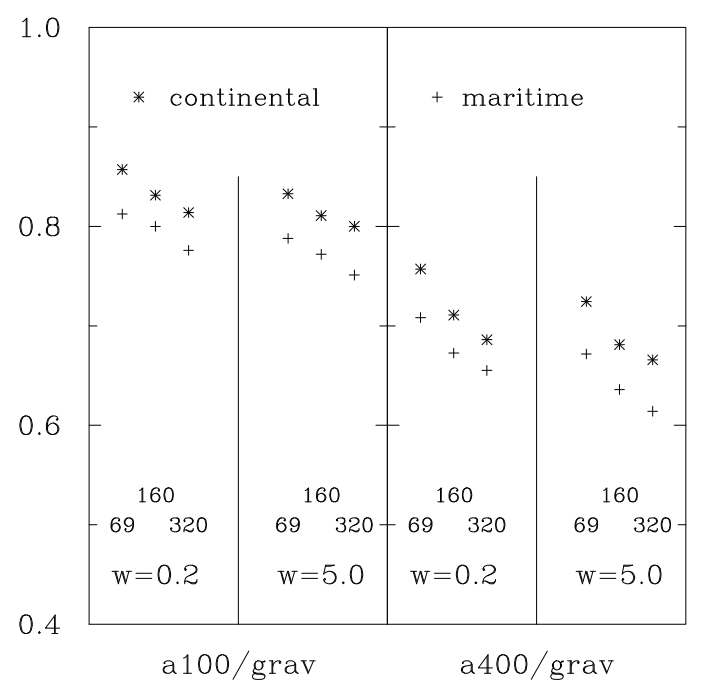

Fig. 14. Ratios between the radar reflectivity transition times for A100 (left panel) or A400 (right panel) and the Hall gravitational kernel for the parcel vertical velocity of 0.2 and $5 \mathrm{~m} \mathrm{~s}^{-1}$ and for grids with $\mathcal{N}=69,160$, and 320 .

0.75 and 0.85 for A100 kernel, and between 0.60 and 0.75 for the A400 kernel.

\section{Discussion and conclusions}

This paper discusses the impact of small-scale cloud turbulence on the development of drizzle and rain using an idealized framework of the adiabatic rising parcel. This study extends that of XWG08 where only collisional growth was considered. Here, activation of cloud droplets and their diffusional growth were added, which allowed studying the impact of different collection kernels in a more realistic framework. Current study represents an intermediate step toward the evaluation of the impact of cloud turbulence using a dynamic cloud model. Results applying two formulations of the gravitational collection kernel were compared to results with turbulent kernels for eddy dissipation rates of 100 and $400 \mathrm{~cm}^{2} \mathrm{~s}^{-3}$. Various grid resolutions in the radius space were used.

The number of bins applied in the simulations had a significant impact on the model results, with lower number of bins resulting in a more rapid development of drizzle and rain. This was shown to be a combination of three effects, as illustrated in Fig. 15. First, the width of the spectrum immediately after the activation (i.e., after the maximum supersaturation) significantly depends on the number of bins applied, with lower number of bins resulting in wider activation spectra. To the authors' knowledge this significant aspect has not been noticed previously despite the fact that such a simple and computationally efficient approach is often used in bin
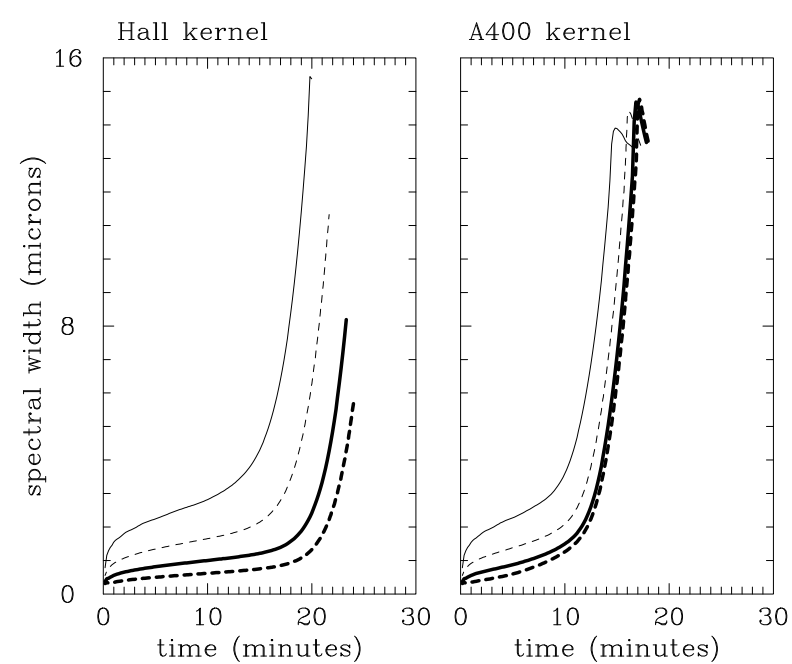

Fig. 15. Evolutions of the spectral width for the MARITIME case, $w=1 \mathrm{~m} \mathrm{~s}^{-1}$ and (left panel) the Hall gravitational kernel and (right panel) the A400 kernel. Thin solid, thin dashed, thick solid, and thick dashed lines are for simulation with $\mathcal{N}$ of $40,80,160$, and 320 , respectively.

microphysics models. Second, representation of diffusional growth suffers from numerical widening of the spectra when the number of bins for radii below, say, $20 \mu \mathrm{m}$ is small. This is why the spectral width is the largest for simulations with low number of bins in Fig. 15. Application of even less diffusive 1D advection scheme to represent diffusional growth of cloud droplets might help to mitigate this problem. However, the fact that the spectral width just after activation is unrealistically small when the number of bins is large (cf. Fig. 15) suggests that the approach used here to represent activation and subsequent diffusional growth may never converge ${ }^{5}$. Arguably the numerical widening helps to initiate and accelerates the autoconversion phase of the collisional growth (the transition from gradual to more rapid increase of the spectral width is also a sign of precipitation formation, see Figs. 4 and 6). This transition happens earlier in simulations with low number of bins as illustrated in Fig. 15, which is consistent with results discussed in Berry and Reinhardt (1974b). Finally, small number of bins can also affect the transition to the accretion phase, when both cloud droplets and drizzle drops coexist. However, for the collisional growth, simulations applying 300 bins should result in solutions that no longer depend on the number of bins (see a discussions in Tzivion et al., 1999 and Wang et al., 2007). As Fig 15 documentss, the effect of small-scale turbulence is also to increase the rate of growth of the spectral width during the initial

\footnotetext{
${ }^{5}$ Note that inserting activated droplets into the first bin and then advecting the spectral density function to represent diffusional growth is analogous to the advection of the delta function, an illposed problem.
} 
diffusion-dominated regime (i.e., within the first $10 \mathrm{~min}$, before the reflectivity reaches $-10 \mathrm{dBz}$ ).

Despite these numerical issues, the estimate of the turbulent speedup factor, the ratio between the rain initiation times for turbulent and gravitational kernels, appears to be only weakly dependent on the number of bins applied in numerical simulations. It also depends weakly on the vertical velocity of the adiabatic parcel and characteristics of the CCN. The speedup factor is between 0.75 and 0.85 for the turbulence intensity of $100 \mathrm{~cm}^{2} \mathrm{~s}^{-3}$ and and between 0.60 and 0.75 for $400 \mathrm{~cm}^{2} \mathrm{~s}^{-3}$. Results presented here show smaller accelerations of rain initiation than in XWG08. This is expected because current calculations include not only collisional growth, but also droplet activation and the diffusional growth. Since collisional growth is inefficient for droplet radii smaller than $10 \mu \mathrm{m}$, considerable time is spent with mostly diffusional growth before autoconversion phase of the collisional growth is initiated.

Theoretical considerations presented in this paper are difficult to relate to processes in natural clouds for several reasons. First, shallow convective clouds, such as cumulus and stratocumulus are strongly diluted by entrainment (e.g., Blyth, 1993; Wang and Albrecht, 1994; Moeng, 2000; Siebesma et al., 2003) and the adiabatic parcel model provides an oversimplified representation of microphysical processes in such clouds. Entrainment has an important and still poorly understood effect on the spectra of cloud droplets. It has been argued to significantly widen the spectra, for instance, through the mixing of parcels with different degree of dilution and thus different reductions of the liquid water content and the mean droplet size. This is why applying an entraining parcel model to quantify the acceleration of the warm rain initiation would not be a robust approach either. Moreover, since the number of activated droplets depends on the strength of the cloud-base updraft, and the updraft can vary significantly across the cloud base, mixing of adiabatic parcels above the cloud base can result in some widening of the adiabatic spectra as well. All these factors result in cloud droplet spectra that are typically significantly wider than those predicted by the adiabatic parcel model (e.g., Brenguier and Chaumat, 2001 and references therein; Pawlowska et al., 2006). One can also argue that the combination of adiabatic water contents and relatively high levels of cloud turbulence considered here is not realistic either. There seems to be considerable evidence from in-situ aircraft observations that high turbulence is typically found in mixing regions where the liquid water contents are considerably below adiabatic. Finally, once formed, drizzle and rain drops have appreciable terminal velocities and they fall out of the parcel before the reflectivity reaches 10 or $20 \mathrm{dBz}$ level. The latter is why the two-dimensional kinematic framework is perhaps more appropriate as argued in Morrison and Grabowski (2007). Nevertheless, the adiabatic parcel model clearly demonstrates that turbulent collisions can accelerate the development of rain significantly.
One of the goals of this study was to access the number of bins required in the bin microphysics framework before such an approach is used in a dynamic cloud model, such as the large-eddy simulation (LES) model, to study with confidence rain formation processes in shallow tropical convection (e.g., in RICO clouds, see Rauber et al., 2007). Although the number of bins seems quite large, one can argue that part of the problem is related to the simplicity of the droplet activation scheme that resulted in unrealistically narrow droplet spectra shortly after activation. Overall, the dependence of the width of the droplet spectra after activation on the number of bins is unexpected. As shown in this paper (and in many previous studies), such a simple scheme results in a realistic prediction of the total number of activated droplets. To predict the width of the activated spectrum, however, a modified approach is needed, for instance, assuming the activation spectrum as in Kogan (1991, Sect. 2b1). Activation of cloud droplets is a difficult problem because it involves detailed physical and chemical characteristics of aerosol particles and, to be treated with confidence, it requires model grid reaching sizes well below $1 \mu \mathrm{m}$ and significant number of bins (see, for instance, the discussion in Srivastava, 1991). However, extending the model grid into droplets significantly smaller than $1 \mu \mathrm{m}$ requires extremely small time steps and most likely cannot be considered for a dynamic model. Moreover, limitations due to spatial resolution of the cloud model need to be considered as well (see discussion in Sect. 4 of Morrison and Grabowski, 2008). We are currently investigating alternative approaches to represent droplet activation in a bin microphysics model of the type used here. The overall goal is to predict activation spectra similar to those obtained in high resolution droplet activation models and in observations. Results of this investigation as well as investigations of rain development and its acceleration due to cloud turbulence using a cloud model with bin microphysics will be reported in future publications.

Acknowledgements. This work was supported by the National Science Foundation (NSF) under grants ATM-0730766 and ATM0527140. WWG acknowledges additional support from NOAA grants NA05OAR4310107 and NA08OAR4310543 as well as DOE ARM grant DE-FG02-08ER64574. LPW also acknowledges support by the NSF Grant PHY99-07949 through Kavli Institute for Theoretical Physics at UCSB, and by National Natural Science Foundation of China (Project No. 10628206). Comments on the manuscript by Charlie Knight, Hugh Morrison, and Knut von Salzen are acknowledged. The National Center for Atmospheric Research is sponsored by the National Science Foundation.

Edited by: A. Nenes 


\section{References}

Ayala, O., Rosa, B., Wang, L.-P., and Grabowski, W. W.: Effects of turbulence on the geometric collision rate of sedimenting droplets: Part 1. Results from direct numerical simulation, New J. Phys., 10, 075015, doi:10.1088/1367-2630/10/7/075015, 2008a.

Ayala, A., Rosa, B., and Wang, L.-P.: Effects of turbulence on the geometric collision rate of sedimenting droplets: Part 2. Theory and parameterization, New J. Phys., 10, 075016, doi:10.1088/1367-2630/10/7/075016, 2008b.

Beard, K. V: Terminal velocity and shape of cloud and precipitation drops aloft, J. Atmos. Sci., 33, 851-864, 1976.

Berry, E. X. and Reinhardt, R. L.: An analysis of cloud drop growth by collection: Part I. Double distributions, J. Atmos. Sci., 31, 1814-1824, 1974.

Berry, E. X. and Reinhardt, R. L.: An analysis of cloud drop growth by collection: Part II. Single initial distributions, J. Atmos. Sci., 31, 1825-1831, 1974.

Blyth, A. M.: Entrainment in cumulus clouds, J. Appl. Meteor., 32, 626-640, 1993.

Bott, A.: A flux method for the numerical solution of the stochastic collection equation, J. Atmos. Sci., 55, 2284-2293, 1998.

Brenguier, J.-L. and Chaumat, L.: Droplet spectra broadening in cumulus clouds. Part I: Broadening in adiabatic cores framework, J. Atmos. Sci., 58, 628-641, 2001.

Chun, J., Koch, D., Rani, S. L., Ahluwalia, A., and Collins, L. R.: Clustering of aerosol particles in isotropic turbulence, J. Fluid Mech., 536, 219-251, 2005.

Clark, T. L.: On modelling nucleation and condensation theory in Eulerian spatial domain, J. Atmos. Sci., 31, 2099-2117, 1974.

Clark, T. L. and Hall, W. D.: A numerical experiment on stochastic condensation theory, J. Atmos. Sci., 36, 470-483, 1979.

Cooper, W. A.: Effectd of variable droplet growth histories on droplet size distribution. Part I: Theory, J. Atmos. Sci., 46, 13021311, 1989.

Falkovich, G., Fouxon, A., and Stepanov, M. G.: Acceleration of rain initiation by cloud turbulence, Nature, 419, 151-154, 2002.

Ghosh, S., Davila, J., Hunt, J. C. R., Srdic, A., Fernando, H. J. S., and Jonas, P.: How turbulence enhances coalescence of settling particles with applications to rain in clouds, Proc. Roy. Soc. London, 461A, 3059-3088, 2005.

Grabowski, W. W. and Vaillancourt, P. A.: Comments on "Preferential Concentration of cloud droplets by turbulence: Effects on the early evolution of cumulus cloud droplet spectra" by Shaw et al., J. Atmos. Sci., 56, 1433-1436, 1999.

Grabowski, W. W.: Numerical experiments on the dynamics of the cloud-environment interface: small cumulus in a shear-free environment, J. Atmos. Sci., 46, 3513-3541, 1989.

Hall, W. D.: A detailed microphysical model within a twodimensional framework: Model description and preliminary results, J. Atmos. Sci., 37, 2486-2507, 1980.

Kogan, Y. L.: The simulation of a convective cloud in a 3-D model with explicit microphysics. Part I: Model description and sensitivity experiments, J. Atmos. Sci., 48, 1160-1189, 1991.

Liu, Y. and Daum, P. H.: Spectral dispersion of cloud droplet size distributions and the parameterization of cloud droplet effective radius, Geophys. Res. Lett., 27, 1903-1906, 2000.

Long, A. B.: Solutions to the droplet collection equation for polynomial kernels, J. Atmos. Sci., 31, 1040-1052, 1974.
Moeng, C.-H.: Entrainment rate, cloud fraction, and liquid water path of PBL stratocumulus clouds, J. Atmos. Sci., 57, 36273643, 2000.

Morrison, H. and Grabowski, W. W.: Comparison of bulk and bin warm rain microphysics models using a kinematic framework, J. Atmos. Sci., 64, 2839-2861, 2007.

Morrison, H. and Grabowski, W. W.: Modeling supersaturation and subgrid-scale mixing with two-moment bulk warm microphysics, J. Atmos. Sci., 65, 792-812, 2008.

Paluch, I. R. and Baumgardner, D. G.: Entrainment and fine-scale mixing in a continental convective cloud, J. Atmos. Sci., 46, 261278, 1989.

Pawlowska, H., Grabowski, W. W., and Brenguier, J. L.: Observations of the width of cloud droplet spectra in stratocumulus, Geophys. Res. Lett., 33, L19810, doi:10.1029/2006GL026841, 2006.

Pinsky, M. B. and Khain, A. P.: Turbulence effects on droplet growth and size distribution in clouds - A review, J. Aerosol Sci., 28, 1177-1214, 1997.

Pinsky, M. B. and Khain, A. P.: Effects of in-cloud nucleation and turbulence on droplet spectrum formation in cumulus clouds, Q. J. Roy. Meteor. Soc., 128, 501-533, 2002.

Pinsky, M. B. and Khain, A. P.: Collisions of small drops in a turbulent flow. Part II: Effects of flow accelerations, J. Atmos. Sci., 61, 1926-1939, 2004.

Pinsky, M. B., Khain, A. P., Grits, B., and Shapiro, M.: Collisions of small drops in a turbulent flow. Part III: Relative droplet fluxes and swept volumes, J. Atmos. Sci., 63, 2123-2139, 2006.

Pruppacher, H. R. and Klett, J. D.: Microphysics of Clouds and Precipitation, Kluwer Academic, 954 pp., 1997.

Rauber, R. M. and Coauthors: Rain in Shallow Cumulus Over the Ocean: The RICO Campaign, B. Am. Meteorol. Soc., 88, 19121928, 2007.

Riemer N. and Wexler, A. S.: Droplets to drops by turbulent coagulation, J. Atmos. Sci., 62, 1962-1975, 2005.

Shaw, R. A., Reade, W. C., Collins, L. R., and Verlinde, J.: Preferential concentration of cloud droplets by turbulence: Effects on the early evolution of cumulus cloud droplet spectra, J. Atmos. Sci., 55, 1965-1976, 1998.

Siebesma, A. P. and Coauthors: A large eddy simulation intercomparison study of shallow cumulus convection, J. Atmos. Sci., 60, 1201-1219, 2003.

Simmel, M., Trautmann, T., and Tetzlaff, G.: Numerical solution of the stochastic collection equation - comparison of the Linear Discrete Method with other methods, Atmos. Res., 61, 135-148, 2002.

Smolarkiewicz, P. K.: A fully multidimensional positive definite advection transport algorithm with small implicit diffusion, J. Comput. Phys., 54, 325-362, 1984.

Srivastava, R. C.: Growth of cloud drops by condensation: Effect of surface tension on the dispersion of drop sizes, J. Atmos. Sci., 48, 1596-1605, 1991.

Stevens, B., Feingold, G., Cotton, W. R., and Walko, R. L.: Elements of the microphysical structure of numerically simulated nonprecipitating stratocumulus, J. Atmos. Sci., 53, 980-1006, 1996.

Tzivion, S., Reisin, T. G., and Levin, Z.: A reference numerical solution of the kinetic collection equation (KCE) for evaluating the accuracy of other numerical methods, J. Comput. Phys., 148, 
527-544, 1999.

Twomey, S.: The nuclei of natural cloud formation part II: The supersaturation in natural clouds and the variation of cloud droplet concentration, Pure Appl. Geophys., 43, 243-249, 1959.

Vaillancourt, P. A., Yau, M. K., Bartello, P., and Grabowski, W. W.: Microscopic approach to cloud droplet growth by condensation. Part II: Turbulence, clustering and condensational growth, J. Atmos. Sci., 59, 3421-3435, 2002.

Wang, L. P., Ayala, O., Kasprzak, S. E., and Grabowski, W. W.: Theoretical formulation of collision rate and collision efficiency of hydrodynamically-interacting cloud droplets in turbulent atmospheres, J. Atmos. Sci., 62, 2433-2450, 2005.

Wang, L. P., Ayala, O., Xue, Y., and Grabowski, W. W.: Comments on "Droplets to drops by turbulent coagulation" by Riemer and Wexler, J. Atmos. Sci., 63, 2397-2401, 2006.
Wang, L. P., Xue, Y., and Grabowski, W. W.: A bin integral method for solving the kinetic collection equation, J. Comput. Phys., 226, 59-88, 2007.

Wang, L.-P., Ayala, O., Rosa, B., and Grabowski, W. W.: Turbulent collision efficiency of cloud droplets, New J. Phys., 10, 075013, doi:10.1088/1367-2630/10/7/075013, 2008.

Wang, Q. and Albrecht, B. A.: Observations of cloud-top entrainment in marine stratocumulus clouds, J. Atmos. Sci., 51, 15301547, 1994.

Xue, Y., Wang, L. P., and Grabowski, W. W.: Growth of cloud droplets by turbulent collision-coalescence, J. Atmos. Sci., 65, 331-356, 2008. 